\title{
Excitons in atomically thin 2D semiconductors and their applications
}

DOI 10.1515/nanoph-2016-0160

Received September 27, 2016; revised December 8, 2016; accepted December 20, 2016

\begin{abstract}
The research on emerging layered two-dimensional (2D) semiconductors, such as molybdenum disulfide $\left(\mathrm{MoS}_{2}\right)$, reveals unique optical properties generating significant interest. Experimentally, these materials were observed to host extremely strong light-matter interactions as a result of the enhanced excitonic effect in two dimensions. Thus, understanding and manipulating the excitons are crucial to unlocking the potential of $2 \mathrm{D}$ materials for future photonic and optoelectronic devices. In this review, we unravel the physical origin of the strong excitonic effect and unique optical selection rules in 2D semiconductors. In addition, control of these excitons by optical, electrical, as well as mechanical means is examined. Finally, the resultant devices such as excitonic light emitting diodes, lasers, optical modulators, and coupling in an optical cavity are overviewed, demonstrating how excitons can shape future 2D optoelectronics.
\end{abstract}

Keywords: excitons; two-dimensional materials; optoelectronics; semiconductors.

\section{Introduction}

Excitons are hydrogen-like bound states formed by a negatively charged electron and a positively charged hole due to Coulomb attraction [1]. Typically, excitons result from the photo-excitation in semiconductors, where they exhibit a spectrally narrow linewidth. The exciton's large oscillator strength and enhanced light-matter interaction allow for efficient recombination and emission of light

*Corresponding author: Xiang Zhang, Nanoscale Science and Engineering Center, University of California, Berkeley, CA 94720 , USA, e-mail: xiang@berkeley.edu; and Materials Sciences Division, Lawrence Berkeley National Laboratory, Berkeley, CA 94720, USA Jun Xiao, Mervin Zhao and Yuan Wang: Nanoscale Science and Engineering Center, University of California, Berkeley, CA 94720 , USA
[2]. More interestingly, the nature of the exciton is akin to an atom in a crystal lattice background. The particle has its own internal fine structure with hydrogen-like excited energy states, potentially enabling future excitonic-carrier devices in quantum computation and excitonic circuits. In addition, as excitons are Bosonic particles, they can condensate to form Bose-Einstein condensates and exhibit superfluid-like transport [3, 4]. While excitons clearly provide a rich platform for physics and devices, they are traditionally difficult to utilize. In typical bulk semiconductor crystals such as silicon, the large dielectric screening and small quasiparticle effective mass result in small exciton binding energies, only $\sim 1-10 \mathrm{~s}$ of $\mathrm{meV}$ [5]. Thus, the bound behavior of an exciton is insignificant compared to the thermal fluctuations unless cooled down to low temperatures. In contrast, the bound exciton states often dictate the optical properties of twodimensional (2D) materials such as monolayer transition metal dichalcogenides (TMDCs). The strong Coulomb interaction in low dimensions and reduced dielectric screening, compared to bulk crystals, naturally makes the excitons bound even at room temperature with a binding energy of hundreds of meV. In addition, the TMDCs' electronic “valleys" host the excitons and give rise to selection rules for the excitation and emission of light. The material's two-dimensionality makes the excitons easily tunable, with a number of external stimuli, enabling many 2D photonic devices, such as light-emitting diodes (LEDs) and lasers. In this paper, we review the progress on understanding the physical excitonic properties in 2D materials and the resulting applications from these quasiparticles.

\section{Exciton physics in 2D semiconductors}

The isolation of graphene from bulk crystals of graphite has sparked a huge amount of research effort into the realm of $2 \mathrm{D}$ materials. While graphene has enabled the discovery of novel physics in electronic transport, the 
gapless nature of the crystal makes it less ideal for the study of optical phenomena in 2D crystals [6]. In contrast, layered TMDCs and black phosphorus are semiconductors with band gaps in the visible and near-infrared energies, respectively. Like graphene, the monolayers of these crystals may be prepared using micromechanical exfoliation (the Scotch tape method). In addition, a huge amount of research has developed chemical synthesis means of achieving up to wafer-scale monolayer TMDCs [7]. The ability to reliably create monolayer semiconductors has been instrumental in unearthing the emerging physics and fabrication of 2D devices.

A

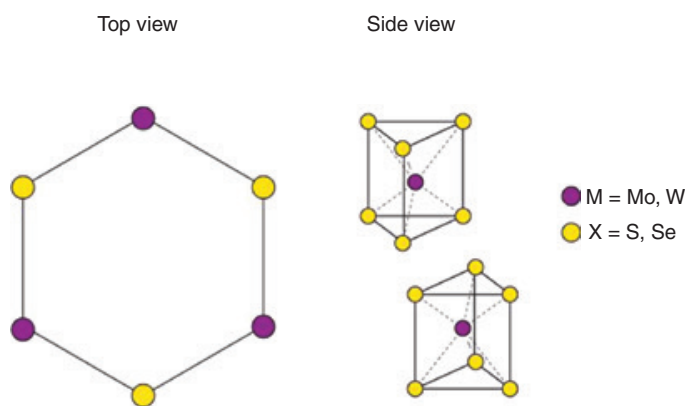

C
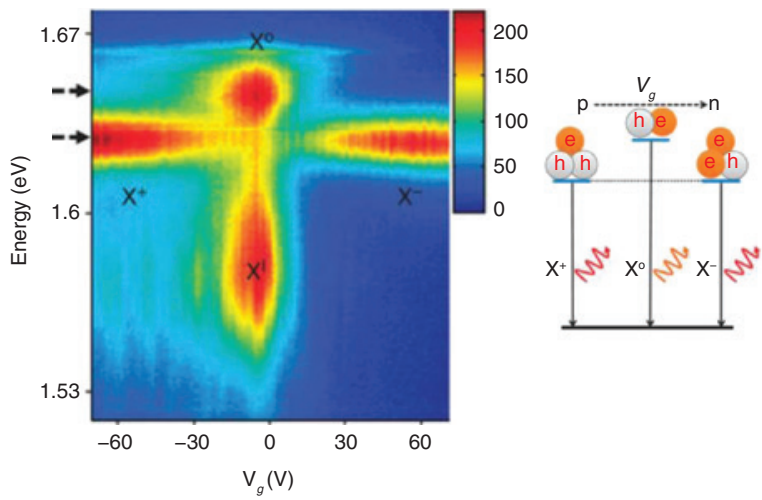

\subsection{Excitons and trions in TMDCs}

The group VIB TMDCs (molybdenum and tungsten based disulfide and diselenides: $\mathrm{MoS}_{2}, \mathrm{MoSe}_{2}, \mathrm{WS}_{2}, \mathrm{WSe}_{2}$ ) are layered van der Waals (vdW) crystals. They are chemically bonded within the layer but only weakly bonded by vdW interlayer interactions between each layer. TMDCs have a trigonal prismatic crystal coordination, with hexagonally arranged transition metals sandwiched between two chalcogen layers [8]. Typically, the crystals studied are of the $2 \mathrm{H}$ phase, which breaks inversion symmetry in a singular layer but restores the symmetry in even numbered layers

B
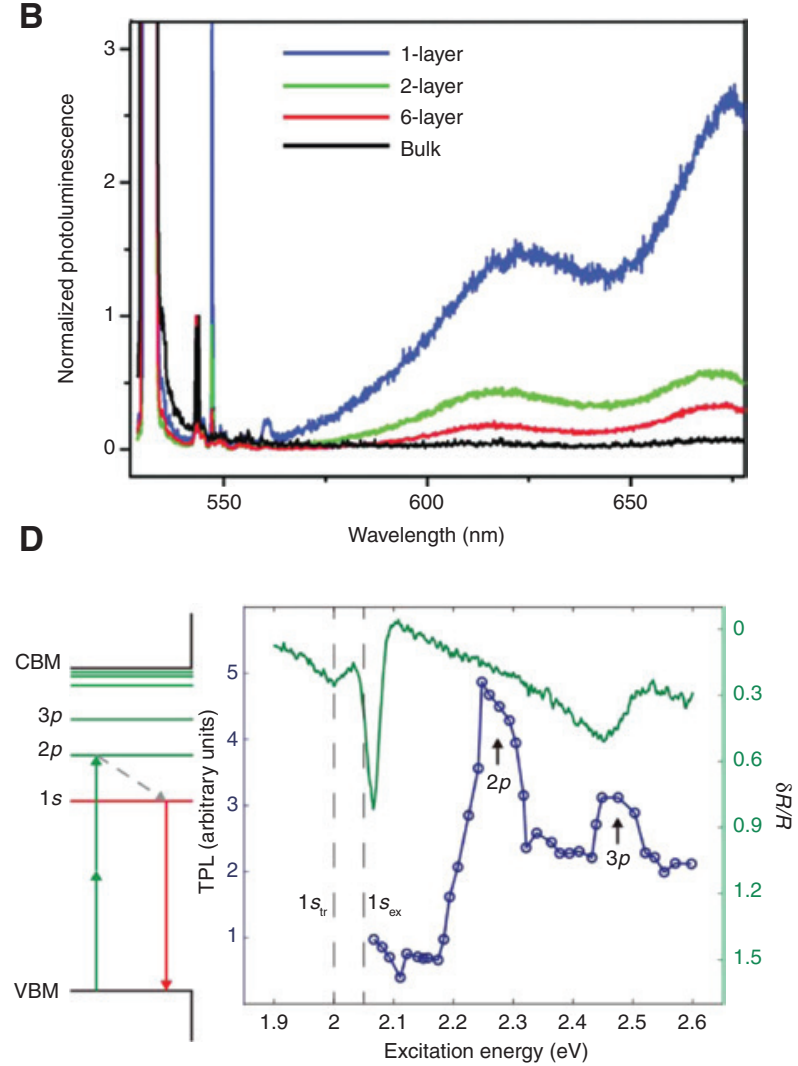

Figure 1: Exciton properties in TMDCs.

(A) Crystal structure in TMDC materials. The top view of the crystal is a hexagonal one, with the actual structure being from trigonal prismatic coordination. Hexagonally arranged transition metals $(M)$ are sandwiched between two layers of chalcogen $(X)$ atoms. The inversion symmetry within one layer is broken but restored upon even layers. (B) Emerging PL from monolayer MoS $_{2}$. The spectra are normalized to the Raman peaks. The PL is much stronger in monolayer than bilayer or greater number of layers. Adapted with permission from [9]. (C) 2010 American Chemical Society. (C) The trion spectra from $\mathrm{MoSe}_{2}$. By tuning the electrical gate $\left(V_{g}\right)$, the PL spectra change from a neutral exciton $\left(\mathrm{X}^{0}\right)$ to a positively $\left(\mathrm{X}^{+}\right)$or negatively charged $\left(\mathrm{X}^{-}\right)$exciton. A schematic is also provided on the right, showing the trions from the gating. The trion binding energy is defined as the energy difference between the trion and the neutral exciton. $X^{\prime}$ denotes the presence of a defect exciton at neutral gating. Adapted with permission from Macmillan Publishers Ltd., Nature Communications [10], (C) 2013. (D) The excitonic energies are given on the left. The green states are those accessible using two-photon excitation, while the red state is the PL, accessed with one-photon excitation. On the right, the TPL spectra are given in blue. The two-photon excitation energy is given by $x$ axis, while the resultant intensity is from the relaxation and emission to the $1 \mathrm{~s}$ state. The green curve is the differential reflectance of the linear absorption, showing that the $2 p$ state has no corresponding linear absorption. Reprinted by permission from Macmillan Publishers Ltd., Nature [11], (c) 2014. 
(Figure 1A). These TMDCs are semiconductors whose bulks have long been known to have an indirect band gap. However, it was discovered that when these crystals are mechanically exfoliated to a monolayer, an indirect-todirect band gap transition occurs due to the lack of any interlayer interactions in the TMDC monolayer. These reduced interlayer interactions manifest themselves as the lowering of the valance band energy, forming a direct band gap with larger absorption and strong photoluminescence (PL) emission compared to the multilayered crystals (Figure 1B) [9]. In $\mathrm{MoS}_{2}$, the PL quantum yield can even approach $100 \%$, with the assistance of chemical treatments [12].

As TMDCs are semiconductors, photo-excitation is expected to generate excitons, pairs of bound electrons, and holes that behave similarly to hydrogen atoms [13]. The recombination of the electron-hole pairs emits a photon that is observed as PL. Within a monolayer, momentumresolved spectroscopy showed that these exciton dipoles are only confined to the in-plane direction [14] with a lifetime in the 100-ps range before recombination [15]. Crucially, these excitons are of particular significance in 2D TMDCs, as the reduced dimensionality causes strong Coulomb interactions due to the spatial confinement and reduced dielectric screening compared to bulk crystals [11]. Hence, it is apparent that the exciton will dominate the observable optical phenomena.

The strong Coulomb interaction was very apparent from the first work done to demonstrate charged excitons, or trions in $\mathrm{MoS}_{2}$ [16]. These trions have been studied in traditional quantum well systems, but they are only observable at low temperatures due to the small $\mathrm{meV}$ binding energy $[17,18]$. The monolayer nature of TMDCs makes it easy to tune the Fermi level and varies the carrier concentration using a field-effect transistor (FET) device [19]. By applying an electric field via the transistor gate, a neutral state can be tuned to be more positive or negative. In $\mathrm{MoS}_{2}$, tuning the Fermi level towards the conduction band results in more electrons. Neutral excitons begin to bind to these extra electrons, and the resultant exciton becomes charged. The recombination of this charged electron also results in PL, although at a lower energy than the neutral exciton. In a similar vein, increasing the hole population by lowering the Fermi level towards the valence band will create positively charged trions, as was observed in $\mathrm{MoSe}_{2}$ (Figure 1C).

Using the difference between the trion luminescent energy and the neutral exciton energy yields the trion binding energy, or the energy to dissociate the extra charge from the neutral exciton. In $\mathrm{MoS}_{2}$, this binding energy is $\sim 20 \mathrm{meV}$, one order larger than the trions observed in quantum wells, resulting in spectral weights which are even significant at room temperature $\left(\mathrm{MoSe}_{2}\right.$ resulted in a similar binding energy of $\sim 30 \mathrm{meV}$ ). This strong Coulomb interaction manifesting as a large trion binding energy points to an even larger exciton binding energy, the energy to dissociate the electron and hole (the energy between the exciton level and the conduction band). This large binding energy is also apparent in the absorption spectrum, as there is an absence of any absorption step that indicates transition to the conduction band [8]. In addition, theoretical estimates put the true quasiparticle band gap over $500 \mathrm{meV}$ above the exciton emission energy, nearly two orders larger than traditional exciton binding energies [20]. Thus, significant effort was put into the elucidation of the true exciton binding energy and quasiparticle band gap.

\subsection{Excitonic binding energy}

As the excitons are hydrogen-like, electron-hole pairs in a Coulomb potential, they form an excitonic series of higher energy states. In TMDCs, each excitonic state has a specific parity that the incident photons can excite. In brief, one-photon states can only excite even parity excitonic states (termed "bright" excitons, such as the PL) and two-photon excitation can only excite odd parity states ("dark" excitons). These two-photon states do not appear in the linear one-photon absorption, therefore, "dark", making it possible to use the two-photon absorption and subsequent bright-state luminescence (two-photon luminescence, TPL) to determine the excitonic structure of TMDCs [11].

In $\mathrm{WS}_{2}$, femtosecond two-photon excitation is used to excite the electrons at the valence band maximum to form excitons. The two-photon excitation energy is scanned, and the bright ("1s" exciton state) luminescence allows for observation of when the two-photons are on resonance with the dark excitonic states. Mapping out the full spectra clearly shows two new peaks in the two-photon excitation from 2.1 to $2.6 \mathrm{eV}$, corresponding to two higher energy dark states, the " $2 p$ " and " $3 p$ " states (Figure 1D). When the twophoton energy is close to the bright exciton, TPL is close to zero, and conversely, one-photon absorption is negligible near the excitonic dark states, as the parity of these states does not allow for one-photon absorption.

Using the $a b$ initio GW method, the quasiparticle band structure of $\mathrm{WS}_{2}$ can be calculated to determine the excitonic states. Calculations show that the calculated $2 p$ and $3 p$ energies match well with experimental energies and show that the excitons are of Wannier nature with an 
in-plane radii are much larger than the dimensions of a unit cell. More interestingly, it was found that the excitons here have significant differences compared to a 2D hydrogen model. In the hydrogen model, orbitals with the same principal quantum number are degenerate in energy (i.e. $E_{3 \mathrm{~s}}=E_{3 \mathrm{p}}=E_{3 \mathrm{~d}}$ ), but the calculations in $\mathrm{WS}_{2}$ show that the higher orbital angular momentum are of lower energy (i.e. $E_{3 \mathrm{~d}}<E_{3 \mathrm{p}}<E_{3 \mathrm{~s}}$ ), due to a phenomenon that is known as the dielectric confinement effect or the anti-screening effect. Finally, the calculations show that the electronic band gap is approximately $2.7 \mathrm{eV}$, with an exciton binding energy of approximately $600-700 \mathrm{meV}$.

Similar one-photon measurements to map the bright excitonic states have shown that the binding energy is $\sim 300 \mathrm{meV}$ [21] and scanning-tunneling-spectroscopy study yielded a binding energy of $550 \mathrm{meV}$ [22]. Clearly, there is a discrepancy in the binding energy of the excitons, perhaps arising from the crystal sources, but this can be approximated at $500 \mathrm{meV}$. For perspective, the binding energy of Wannier excitons is $\sim 10 \mathrm{meV}$ in traditional semiconductors [23]. The large binding energy in TMDCs is a result of the strong Coulomb interaction from the monolayer, large effective carrier masses, and weak dielectric screening. More recently, the full excitonic structure has been revealed through the inter-excitonic transitions using terahertz spectroscopy, unraveling the rich exciton physics in these materials [24].

\subsection{Valley excitons and selection rules}

The excitonic nature of TMDCs is of particular significance due to the material's promise in "valleytronics", a concept spawning from grapheme [25]. In graphene, the crystal momentum space's Brillouin zone is hexagonal. At the corners, termed the $\mathrm{K}$ points, are electronic "valleys", a degree of freedom that is akin to charge and spin. Graphene hosts two sets of valleys at the $\mathrm{K}$ and $-\mathrm{K}$ (also referred as $\mathrm{K}^{\prime}$ ) points. The possibility to control these valleys in devices to act as information carriers is termed valleytronics.

However, graphene has had difficulty in generating a valley "current," requiring configurations such as bilayer domain walls [26], specific crystalline edges [27, 28], or strain [29]. Theoretically, it can be shown that the generation of a valley polarization to differentiate the $\pm \mathrm{K}$ valleys requires the breaking of inversion symmetry in the crystal. In brief, this requirement yields a Berry curvature $(\Omega)$ and orbital magnetic moment $(\mathrm{m})$, allowing $\mathrm{K}$ and $-\mathrm{K}$ to take on opposite values. In contrast, inversion symmetric crystals like graphene, $\Omega$ and m must take on the same values under inversion, thus $\mathrm{K}$ and $-\mathrm{K}$ become indistinguishable. Hence, achieving a valley polarization requires a crystal system with broken inversion symmetry and two distinguishable valleys in the crystal momentum space [30, 31].

The hexagonal nature of TMDCs and broken inversion symmetry of the monolayers makes them an ideal system to realize valley polarization. The presence of spin-orbit coupling and time reversal symmetry splits the bands at the $\mathrm{K}(-\mathrm{K})$ points with the opposite spins (Figure 2A). Early calculations showed that photo-excitation is able to selectively excite the $\pm \mathrm{K}$ valley electrons from the spinpolarized bands with circularly polarized light. Experimentally, three independent works demonstrated this valley-dependent circular dichroism through excitation of the K $(-\mathrm{K})$ valley with $\sigma-(\sigma+)$ light to yield PL of the same polarization in $\mathrm{MoS}_{2}$ (Figure 2B). Thus, valley polarization is achieved through the exciton selection rules in TMDCs [13, 32, 34].

The parity selection from TPL indicates that there are selection rules that govern valley excitations, which include nonlinear excitations. As the angular momentum of the system must be conserved, it is possible to easily summarize the selection rules as follows (schematically in Figure 2C) [33]:

$$
\Delta m \hbar=\Delta \tau \hbar+\Delta l \hbar+3 N \hbar .
$$

Upon absorption of incident light, the spin angular momentum of the photons, $\Delta m \hbar$, where $m= \pm 1$ for $\sigma \pm$ light, changes the angular momentum of the electrons in the valley $(\Delta \tau \hbar$, where $\tau=+1(-1)$ for the $-\mathrm{K}(+\mathrm{K})$ valley, the angular momentum of the excitons (via the excitonic quantum number $l$ ), and the change in the angular momentum of the crystal lattice (3Nh, resulting from the symmetry of the crystal). One can easily verify that the one-photon excitations to the 1s excitonic level $(l=0)$ simply yield excitation of K $(-\mathrm{K})$ valley with $\sigma-(\sigma+)$ light.

$$
( \pm 1) \hbar=( \pm 1) \hbar+(0) \hbar+3(0) \hbar
$$

For nonlinear light, one can consider the second harmonic generation (SHG). The broken inversion symmetry of the monolayer results in finite $\chi^{(2)}$ and strong secondorder nonlinear effects. This SHG has been able to map out the grain boundaries in chemically grown $\mathrm{MoS}_{2}$ as well as distinguish the nonlinear differences of the chemically grown edges [35]. In case of resonant 1s SHG, two photons are pumped at the energy of the 1s exciton level. Using incident $\sigma+$ light $(m=1)$, one can observe that this can only be satisfied with excitation to the K valley $(\tau=-1)$ and have resultant $\sigma-$ emission: 
A
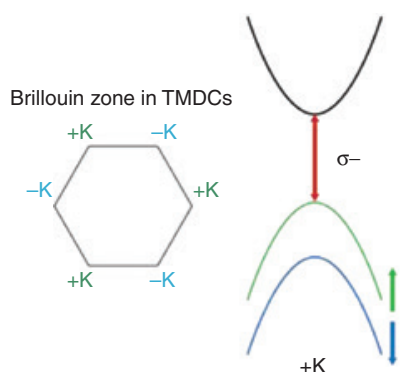

$+\mathrm{K}$

C $\mathrm{CBC}$

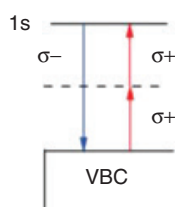

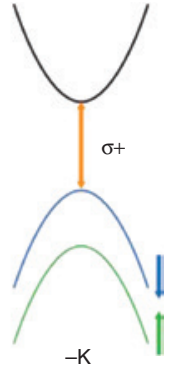

B

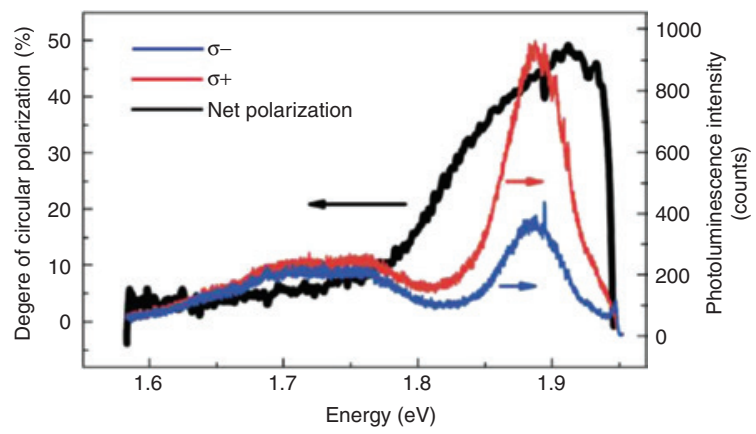

E
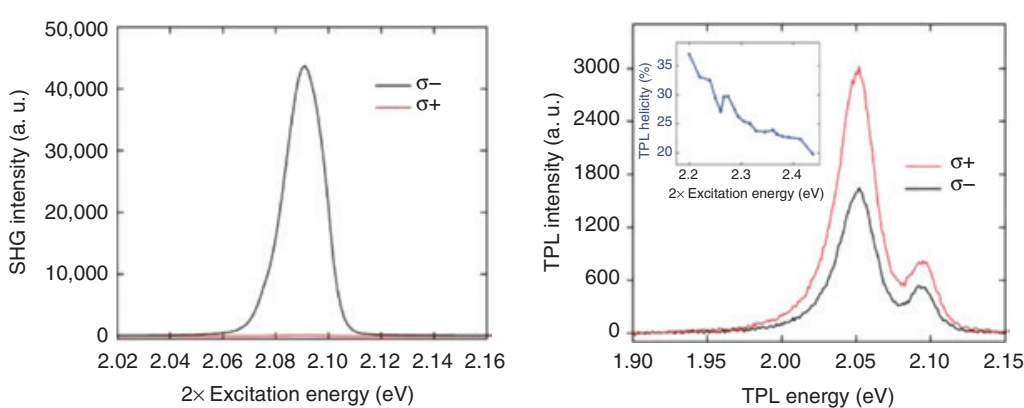

Figure 2: Excitonic selection rules in TMDCs.

(A) Simplified picture of the electron bands near the K points of the Brillouin zone. TMDCs also have a hexagonal Brillouin zone like graphene with the band gaps occurring at the K points. The $d$-orbitals cause spin-orbit interactions, which spin polarize and split the valence bands. To preserve time-reversal symmetry, the spin polarization of the K valley valence band must be the opposite of the $-\mathrm{K}$ valley. Here, green indicates the spin-up valence band, while blue indicates the spin-down valence band at the band edges. (B) Circular dichroism in $\mathrm{MoS}_{2}$. Using $\sigma+$ light to photo-excite the electrons in $\mathrm{MoS}_{2}$, there is a preference for the emission of $\sigma+$ light. This result shows a circular dichroism or valley polarization of approximately $50 \%$. Adapted with permission from Macmillan Publishers Ltd., Nature Communications [32], ( ) 2013. (C) Schematic showing the selection rules for nonlinear two-photon excitations at the +K valley. For SHG (on the left), two photons with opposite polarization emit a photon corresponding to the valley. This is due to the virtual nature of the SHG process and the crystal momentum contribution. As for TPL (on the right), the same polarization of light as the input is observed. (D) SHG selection rule evidence using $\sigma+$ light to excite WS , results only in $\sigma$ - emission from the $+\mathrm{K}$ valley. (E) Experimental evidence of the TPL process preserving the valley polarization. The same excitation of $\sigma+$ is used and a polarization of $>35 \%$ can be achieved. (C-E) Adapted with permission from Macmillan Publishers Ltd., Light: Science \& Applications [33], @ 2015.

$$
2(+1) \hbar=-1 \hbar+0 \hbar+3(1) \hbar
$$

Thus, if the exciton's angular momentum does not change, the SHG process must generate light with opposite circular polarization (Figure 2D), achieved by transferring the angular momentum to the crystal lattice. Similarly, in the TPL process, the angular momentum can be transferred to the exciton's higher energy states. If the excitation is resonant with the $2 p$ state with an excitation of $\sigma+$, then

$$
2(+1) \hbar=+1 \hbar+1 \hbar+3(0) \hbar .
$$

The polarization from PL is the same as the incident polarization (Figure 2E), without involving the crystal lattice. Experimentally, these selection rules have been verified showing that SHG produces light of opposite circular polarization to the incident photons while the TPL process will preserve the circular polarization $[33,36]$.

Elucidating the selection rules for linear and nonlinear optical processes allows for the full prediction and control of the valley excitations in these TMDC crystals and has potential to enable valleytronic devices based on photon polarizations.

\subsection{Black phosphorus: a case of anisotropy}

Black phosphorus is a vdW crystal that first garnered significant attention due to its high carrier mobility for semiconductor devices [37,38]. Black phosphorus and respective monolayer phosphorene lack the same electronic valleys in graphene and TMDCs, but the 2D nature 
also results in the same strong Coulomb confinement that strongly binds its excitons. Phosphorene, like monolayer TMDCs, also exhibits a direct band gap but retains a direct, albeit smaller, band gap in the multilayered and bulk forms.

More interestingly, in black phosphorous, each phosphorus atom is covalently bonded to three adjacent atoms to form a buckled honeycomb lattice, resulting in strong in-plane anisotropy (Figure 3A). Theoretical calculations showed that excitonic nature is only observable when the incident light is polarized along the armchair direction of the crystal. Phosphorene, in particular, strongly absorbs armchair polarized light between $1.1 \mathrm{eV}$ and $2.8 \mathrm{eV}$ but is completely transparent to the zigzag polarization, making phosphorene a natural linear polarizer. Anisotropic absorption suggests that exciton wave functions are also heavily anisotropic, with early calculations showing that the excitons are spread along the armchair direction of the crystal [40].
Experimentally, phosphorene was isolated and polarization-resolved PL was able to demonstrate that the excitonic nature of the emission was heavily in the armchair direction (Figure 3B). Comparatively, phosphorene is a $2 \mathrm{D}$ crystal whose excitonic properties are similar to one-dimensional carbon nanotubes [39]. The excitons of phosphorene also exhibit a large binding energy, once again due to the strong Coulomb interaction in two dimensions. Photoluminescence excitation intensity maps show that the strongest PL intensity (at $1.3 \mathrm{eV}$ ) occurs when excitation is $\sim 2.4 \mathrm{eV}$ (Figure 3C). Like carbon nanotubes, the absorption of phosphorene increases dramatically near the quasiparticle band edge, thus the exciton binding energy can be extracted to be approximately $900 \mathrm{meV}$.

The addition of phosphorene to the 2D family enriches the optical responses found, adding an even larger binding energy and a layer of anisotropy to the rich exciton properties, which can be explored in these spatially confined crystals.
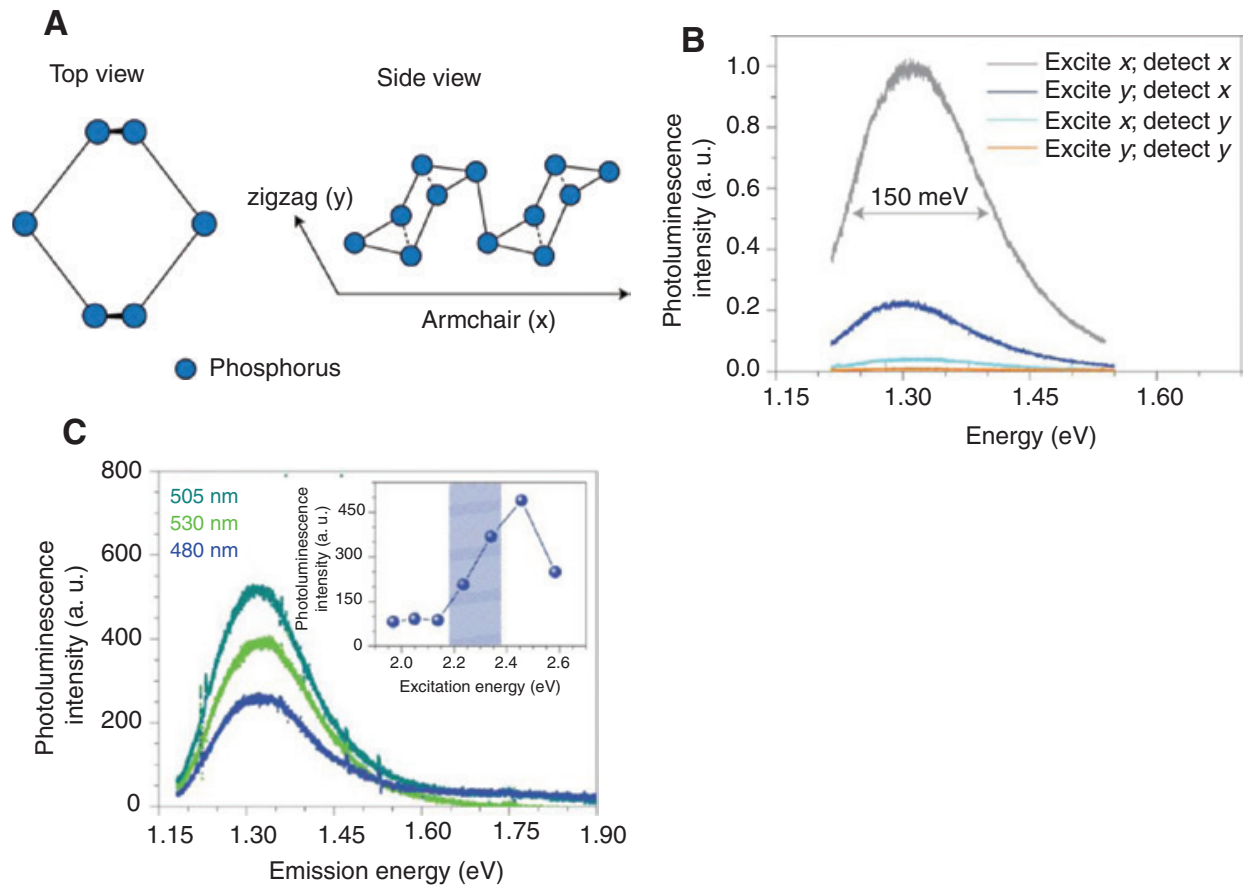

Figure 3: Anisotropic excitons of phosphorene.

(A) Crystal structure of monolayer black phosphorus, phosphorene. The top view presents a buckled honeycomb lattice, with the side view showing the anisotropic nature of the crystal. These units are repeated along the zigzag direction, while the armchair direction shows two units for clarity. (B) Anisotropic excitons of phosphorene. Exciting $x$ (armchair direction) and $y$ (zigzag direction) give very different optical responses. Only exciting and detecting the armchair direction gives significant $\mathrm{PL}$, while exciting and detecting the zigzag direction gives no PL. (C) Binding energy of phosphorene determined using higher energy excitation. Near the band edge, the PL increases dramatically, as shown in the inset's shaded blue region. This indicates the approximate energy of the quasiparticle gap. (B-C) Adapted with permission from Macmillan Publishers Ltd., Nature Nanotechnology [39], ๔ 2015. 


\section{Exciton tunability in 2D semiconductors}

A fundamental requirement for devices is the ability to control desired parameters. For 2D semiconductors, this means ways to manipulate the excitonic behavior and emission. The reduced dimensionality of these semiconductors enables the large tuning of optical properties via external stimuli such as applied electric fields, strain, magnetic fields, and light.

\subsection{Electrical tuning}

Among the first applications of monolayer $\mathrm{MoS}_{2}$ was for transistors. As it is an atomically thin semiconductor, it is possible to use the field effect to electrostatically dope carriers and thus tune the current in a transistor structure. Using the transistor's gate voltage, Mak et al. [16] first observed that tuning the carrier density to high electron populations changes the absorption spectra (Figure 4A) and PL from the exciton to the tightly bound negative trions $\left(\mathrm{X}^{-}\right)$, a quasiparticle composed of two electrons and a hole. The negative trion also possesses large binding energy $(\sim 20 \mathrm{meV})$, such that robust trion PL can be detected at room temperature (Figure 4B). In addition, circularly polarized resolved gate-dependent PL showed helicity from emission of trions, which indicates that such quasiparticles can be optically created with valley and spin-polarized holes. In parallel, Ross et al. [42] investigated the electrostatic gate effect on PL of positively charged $\left(\mathrm{X}^{+}\right)$, neutral $\left(\mathrm{X}^{0}\right)$, and negatively charged $\left(\mathrm{X}^{-}\right)$
A

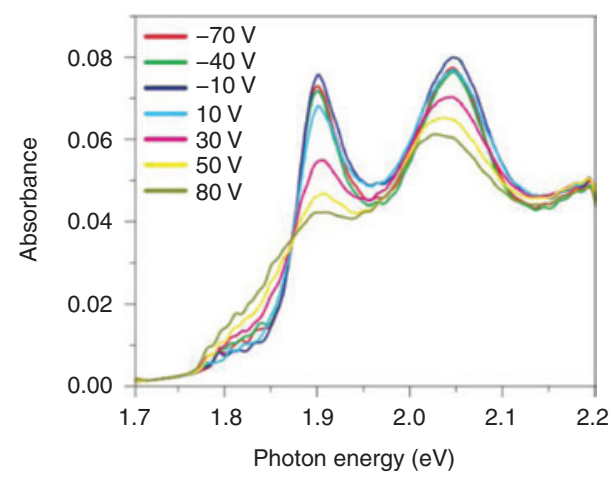

C

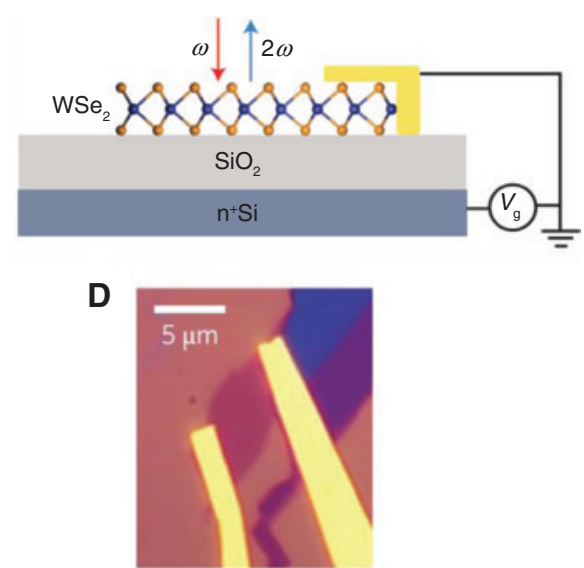

B

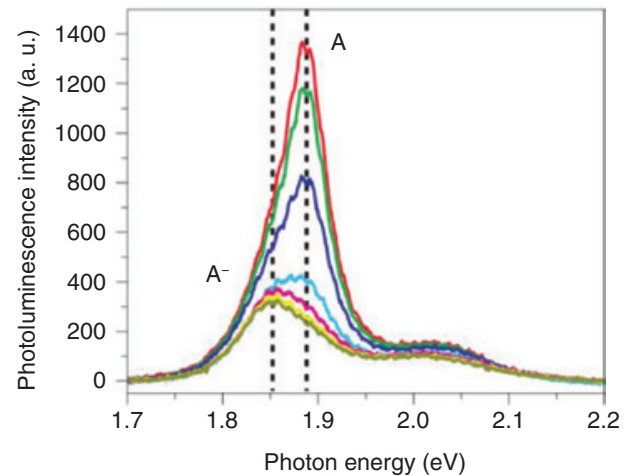

E

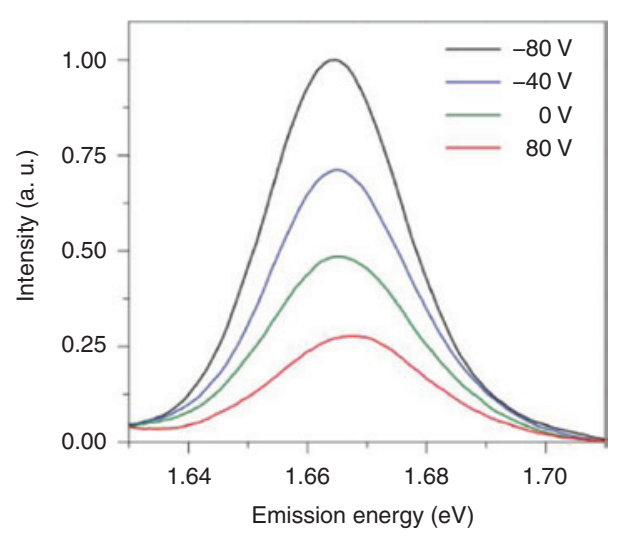

Figure 4: Electrical tuning of excitons.

(A) Monolayer $\mathrm{MoS}_{2}$ absorption spectra at different back-gate voltages in a FET device. The global absorption with significant "A" exciton $(1.92 \mathrm{eV})$ and "B" exciton (2.08 eV) shows amplitude variation, peak shift, and linewidth change under different gate bias. (B) Photoluminescence spectra of same device under different back-gate bias. Here " $\mathrm{A}^{-}$" is the trion. (A-B) Adapted with permission from Macmillan Publishers Ltd., Nature Nanotechnology [16], ( ) 2013. (C) Schematic of a monolayer WSe $e_{2}$ transistor for second harmonic generation. (D) Microscope image of the transistor. The WSe ${ }_{2}$ is the light purple area between two metal electrodes. (E) SHG spectra on "A" exciton resonance under different gate bias at room temperature. The intensity increases about four times when the bias switches from $80 \mathrm{~V}$ to $-80 \mathrm{~V}$. (C-E) Adapted with permission from Macmillan Publishers Ltd., Nature Nanotechnology [41], @ 2015. 
excitons in monolayer $\mathrm{MoSe}_{2}$. Such large binding energy results from the enhanced Coulomb interaction due to the strong confinement and heavy effective masses. Also, they found the extra charge binding energies for $\mathrm{X}^{+}$and $\mathrm{X}^{-}$to be nearly the same, indicating the similar effective mass for electrons and holes, which is consistent with theoretical calculations.

Further quantitative study on gate-dependent exciton binding energy was done by Chernikov et al. [43]. The authors demonstrated continuous tuning of the exciton binding energy in monolayer $\mathrm{WS}_{2}$ under bias in a FET. By measuring differential reflection, the ground and excited excitonic states are tracked as a function of gate voltage. With the density of electrically injected free charge carriers up to $8 \times 10^{12} \mathrm{~cm}^{-2}$, they observed a decrease of the exciton binding energy of about $100 \mathrm{meV}$ due to increasing screening of the Coulomb interaction. Based on their estimation, complete ionization of the excitons can occur at a carrier density of several $10^{13} \mathrm{~cm}^{-2}$.

The exciton's large electrical tunability in oscillation strengths also modifies the nonlinear spectrum. Seyler et al. [41] showed how the electrostatic doping via the transistor gate is able to change the SHG magnitude (Figure 4C,D). The SHG amplitude at the A-exciton resonance varied about four times at room temperature (Figure 4E) and over an order of magnitude at low temperature and nearly a factor of four at room temperature.

All of the above studies revealed the large electrical tunability on excitonic spectroscopy in 2D TMDCs associated with carrier density-dependent many-body Coulomb interactions. The electric field itself was found to not appreciably modulate the dielectric constant in single layers, thus electrical control is more of an access to the monolayer's carrier concentrations [44]. Thus, the exciton's dependence on the carrier concentrations may boost interests on fundamental studies of many-body interactions and lead to chip-scale, electrically tunable optoelectronic and valleytronic applications at the 2D limit.

\subsection{Mechanical tuning}

Mechanical flexibility is a unique advantage for 2D materials $[45,46]$. With mechanical strain, the electronic band structure that is linked with the lattice constant can change accordingly. Therefore, optical properties, especially inter-band transitions, in TMDC are expected to vary. By putting atomically thin $\mathrm{MoS}_{2}$ on flexible substrates, He et al. [47] demonstrated the continuous tuning of the electronic structure when applying a uniaxial tensile strain. As large as $70-\mathrm{meV}$ redshift per percent of applied strain for direct gap transitions has been observed by absorption and PL spectroscopy. A similar observation has also been reported by Conley et al. [48], where uniaxial tensile strain in the range of $0-2.2 \%$ modifies the phonon spectra and electronic band structure of monolayer and bilayer molybdenum $\mathrm{MoS}_{2}$ (Figure 5A,B). Photoluminescence spectroscopy indicates an energy decrease of $45 \mathrm{meV}$ per percent strain for monolayer and $120 \mathrm{meV}$ per percent strain for bilayer $\mathrm{MoS}_{2}$ in the optical band gap (Figure 5C). In addition, given a different strain-dependent response for carriers with different effective mass, a direct-to-indirect transition of the optical band gap accompanies a pronounced decrease in the PL intensity at an applied strain of $\sim 1 \%$. From the Raman spectroscopy, phonon modes soften with increased strain and break the degeneracy in the $\mathrm{E}^{\prime}$ Raman mode of $\mathrm{MoS}_{2}$ [48].

As the strain affects the band structure, it also influences valley polarization. As shown in Figure 5D and E, Zhu et al. [49] demonstrated a strong decrease of PL helicity in both monolayer (from 10\% to 6\%) and bilayer (from 6\% to $0 \%$ ) samples by applying strain up to $0.8 \%$. However, the exact depolarization mechanism induced by strain is not quite clear. The lattice constant change influences multiple aspects such as band structure, phonon spectrum, and exciton exchange interaction, requiring additional study to clarify the exact depolarization process under strain.

\subsection{Magnetic tuning}

Monolayer TMDCs preserve time reversal symmetry, with energetically degenerate excitons in the $\pm \mathrm{K}$ valleys. Thus, valley polarization can only be distinguished by circular dichroism. However, this can be broken using a strong magnetic field by utilizing the Zeeman effect, which shifts the excitonic energy levels in the different valleys and offers further control for valleytronic applications.

Both Aivazian et al. [50] and Srivastava et al. [51] elucidated the influence of magnetic field on the spin, orbital, and valley angular momentums. While the Zeeman shift, due to the spin magnetic moment, does not affect optical resonances, which conserve spin, atomic orbital contribution does play a role. While the conduction band edge is composed of electrons in $\mathrm{d}_{z}{ }^{2}$ with $m=0$, the valence electrons of the $\mathrm{K}$ valley are composed of d-orbitals with $m=-2$ and p-orbitals with $m=-1$ and 
A

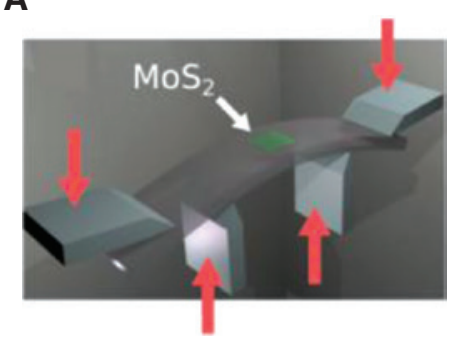

D
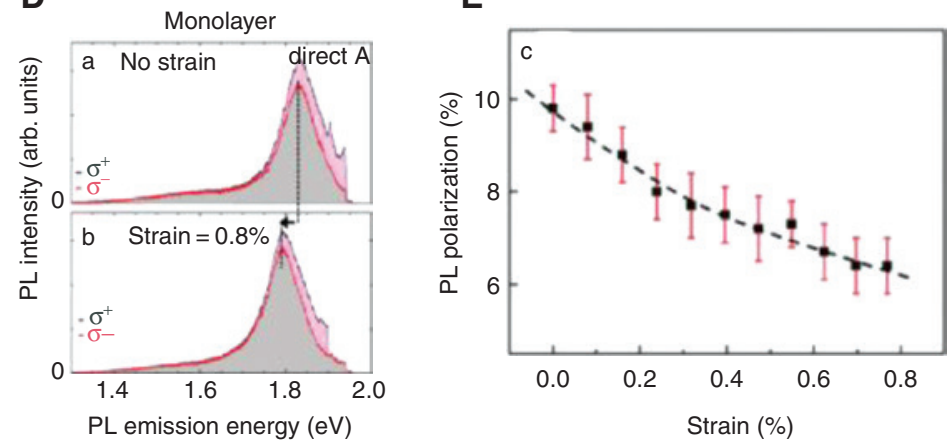

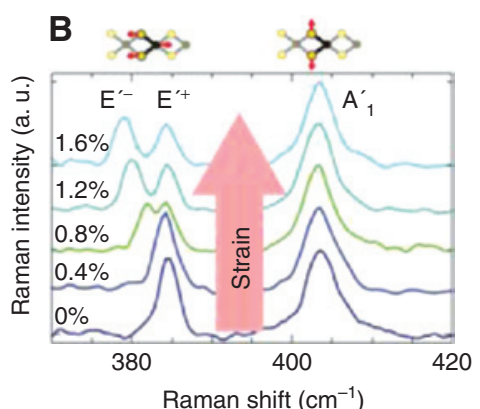

E

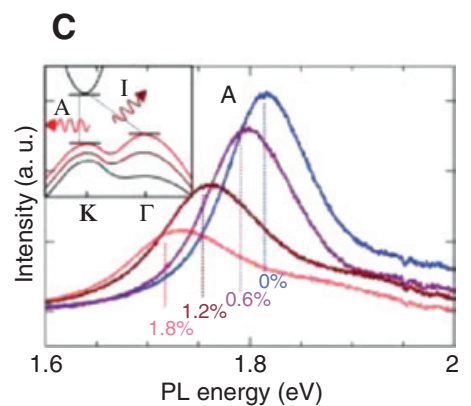

Figure 5: Mechanical tuning of excitons with strain.

(A) Schematic of the bending setup to strain $\mathrm{MoS}_{2}$. Ultrathin $\mathrm{MoS}_{2}$ flakes are clamped with titanium on a SU8/polycarbonate substrate that is strainable. (B) Raman spectrum as function of the in-plane strain in monolayer $\operatorname{MoS}_{2}$. The out-of-plane oscillation $\mathrm{A}_{1 \mathrm{~g}}$ mode shows almost no change under in-plane strain. In contrast, the in-plane oscillation $E_{2 g}$ displays peak shift and peak splitting into two modes with increasing strain. (C) Photoluminescence of strained monolayer $\mathrm{MoS}_{2}$. By applying strain, PL emission intensity decreases and peak position shifts to lower energy. The inset shows the competition between direct and indirect transition under strain $\mathrm{MoS}_{2}$. (A-C) Adapted with permission from [48]. ( 2013 American Chemical Society. (D) PL emission for $\sigma+$ and $\sigma-$ polarization for $\mathrm{MoS}_{2}$ with no strain and $0.8 \%$ in-plane strain. The laser excitation is at $1.95 \mathrm{eV}$ with $\sigma+$ polarization. (E) Valley polarization as function of strain, decreasing as the strain is increased. (D-E) Adapted with permission from [49] @ 2013 APS.

reversed for the $-\mathrm{K}$ valley. Thus, applying an out-ofplane magnetic field leads to no change in the conduction band but a valley-dependent energy shift in the valence band. An additional Zeeman shift is induced by valley magnetic moments associated with intercellular electron motion (Figure 6A). Using polarization-resolved magneto-PL at $30 \mathrm{~K}$, it was confirmed in $\mathrm{WSe}_{2}$ that the $\mathrm{PL}$ from the $-\mathrm{K}$ valley exciton is lowered in energy with respect to the $\mathrm{K}$ valley exciton for $B>0$ and higher in energy for $B<0$ (Figure 6B). The magnetic field induces an excitonic shift of $\sim 0.11 \pm 0.01 \mathrm{meV}$ per Tesla (Figure 6C). Such valley energy degeneracy breaking also affects the valley polarization, as shown in Figure 6D. In addition, there is a striking difference between the magnetic response of the neutral exciton and charged trions, due to the renormalization of excitonic spectrum arising from strong exchange interactions.

Additional work by Li et al. [52] and MacNeill et al. [53] used circularly polarized PL in $\mathrm{MoSe}_{2}$ under magnetic fields up to $10 \mathrm{~T}$. Neutral and charged excitons shift linearly with field strength at a rate of $\mp 0.12-22 \mathrm{meV}$ per Tesla for emission arising from the $\pm \mathrm{K}$ valleys, indicating the lifting of the valley degeneracy.

\subsection{Optical tuning}

In addition to using a magnetic field to lift the valley degeneracy, light can generate a pseudo-magnetic field. Using the optical Stark effect, the TMDC exciton levels can be tuned using intense circularly polarized light. This effect is based on the coherent interaction between an exciton and a photon, understood via Floquet theory [54]. Within this framework, a time-periodic Hamiltonian has quasistatic eigenstates that are evenly spaced in units of the driving photon energy. For a simple illustration (Figure 7A), a two-level atomic system in the presence of monochromatic light is considered. In brief, a two-level atom has eigenstates $|a\rangle$ and $|b\rangle$ that interact with the light's electric field, $E(t)=E_{0} \cos \omega t$ by coherent absorption to form photon-dressed Floquet states, $|\mathrm{a}\rangle \rightarrow|a+\hbar \omega\rangle$ and $|b\rangle \rightarrow|b-\hbar \omega\rangle$. The hybridization of these optically 
A
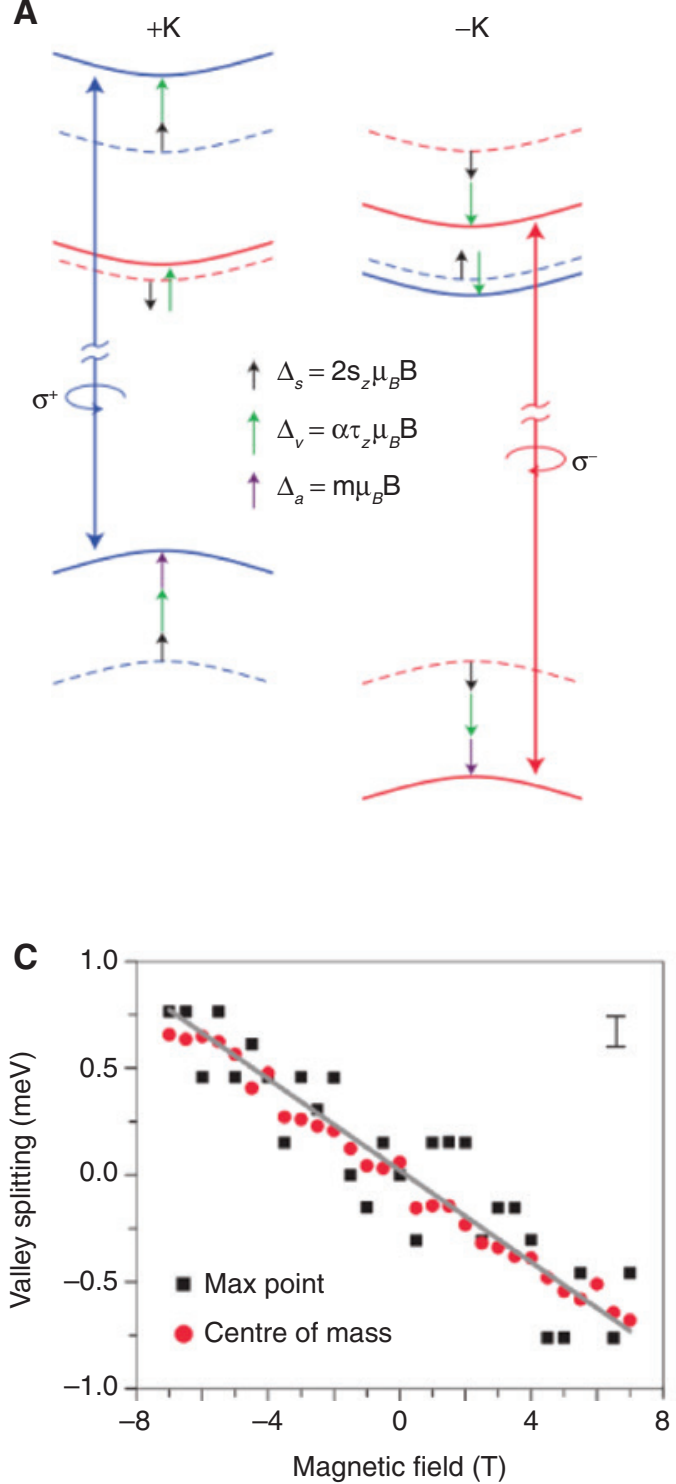

B
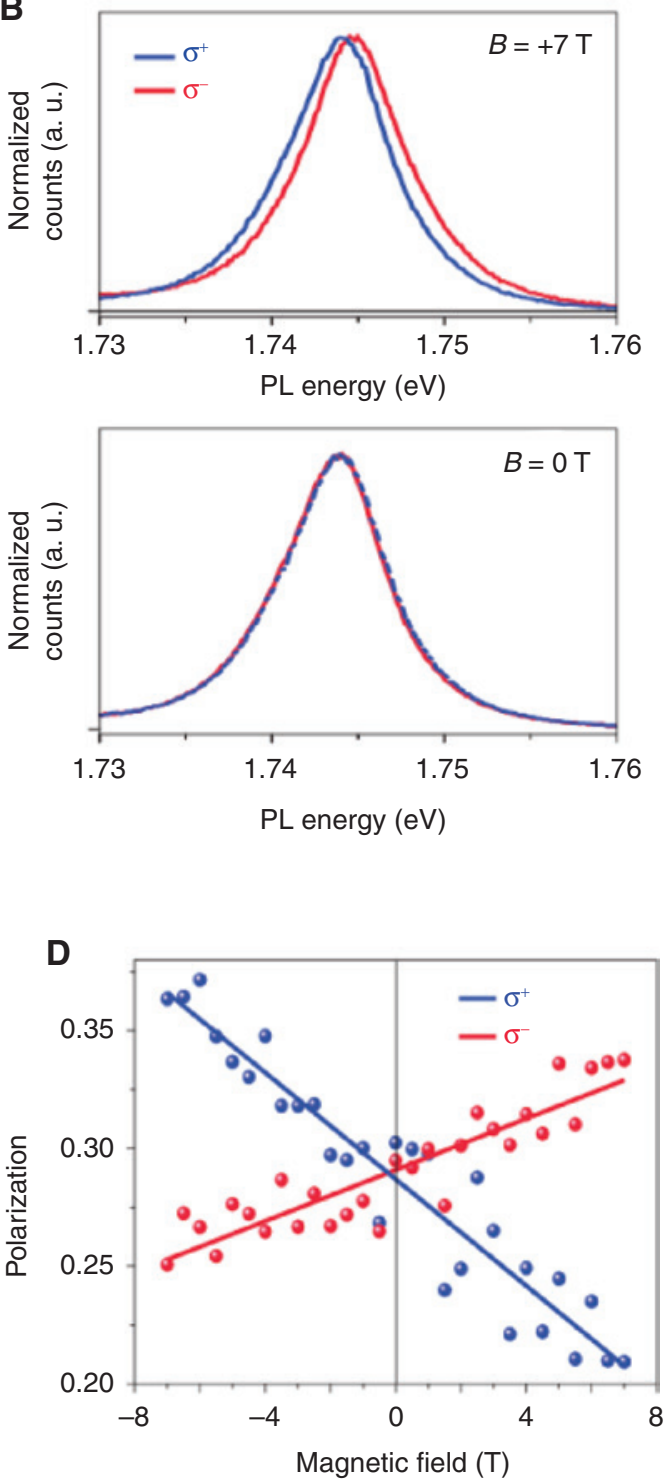

Figure 6: Valley Zeeman effect.

(A) Diagram of $K$ and $-K$ valley energy levels shift under an out-of-plane magnetic field. The valley-dependent Zeeman effect is attributed to three factors (black for spin, green for valley, and purple for atomic orbital). (B) Valley exciton PL with and without a magnetic field. $K$ and $-K$ valley energy degeneracy lifts in the presence of a magnetic field. Blue and red curves correspond to co-polarized excitation and detection. (C) The magnetic field-dependent $K$ and $-K$ valley energy splitting extracted by two methods. The solid line is a linear fit. The bar at the top right is the spectral resolution of measurement system. (D) Valley polarization degree for excitons as a function of field strength. Blue (red) represents $\sigma+(\sigma-)$ pump. (A-D) Adapted with permission from Macmillan Publishers Ltd., Nature Physics [50], ( 2015.

generated Floquet states and equilibrium states results in a shift in energy level separation, with magnetic field given by

$$
\Delta E=\frac{\mathrm{M}_{a b}^{2}\left\langle E(t)^{2}\right\rangle}{\delta},
$$

where $\mathrm{M}_{a b}$ represents a polarization matrix between $|a\rangle$ and $|b\rangle$ and $\delta$ is $\left(E_{b}-E_{a}\right)-\hbar \omega$. The sign of $\Delta E$ is determined by the sign of $\delta$, while magnitude is proportional to incident light intensity and becomes significant near resonant energy levels.

Based on these effects, Sie et al. [54] and Kim et al. [56] independently demonstrated that the optical Stark effect in $\mathrm{WSe}_{2}\left(\mathrm{WS}_{2}\right)$ results in an energy split of more than 10 (18) meV between the $\pm \mathrm{K}$ valleys (Figure 7BD), which corresponds to a pseudo-magnetic field as high as 60 T. Significant absorption change in specific 
A

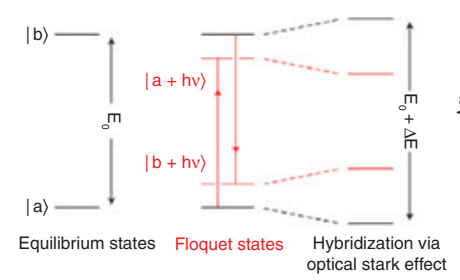

D

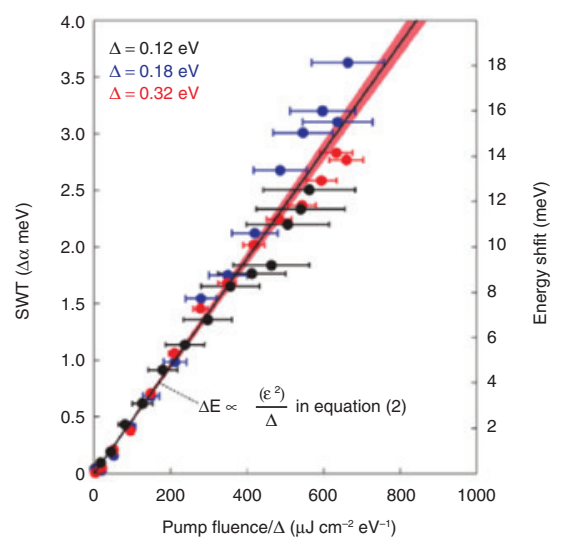

B

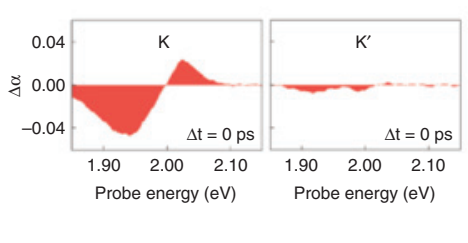

E
C

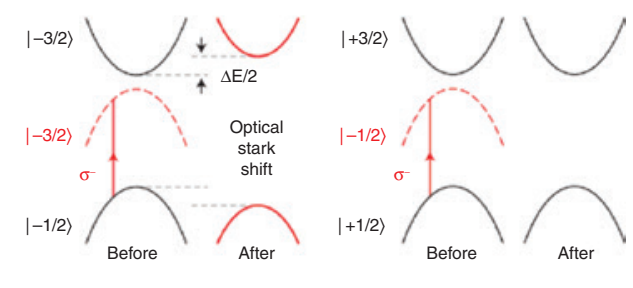

F

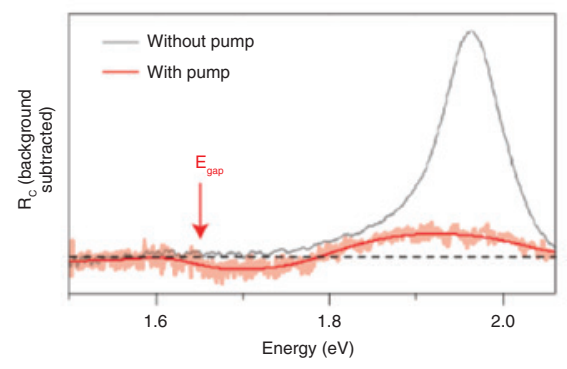

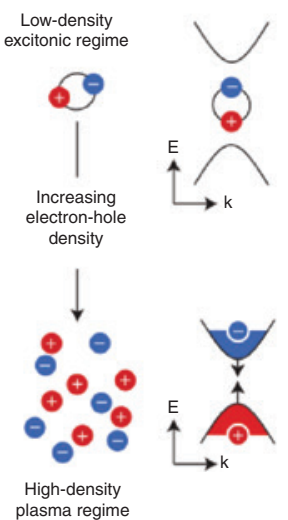

Figure 7: Optical control of excitons.

(A) Illustration of optical stark effect for two-level system. Ground state $|a\rangle$ and excited state $|b\rangle$ can hybridize with Floquet states $|a+\hbar \omega\rangle$ and $|b+\hbar \omega\rangle$ induced by coupling with electromagnetic field, leading to energy shift $\Delta \mathrm{E}$. The magnitude and sign of $\Delta \mathrm{E}$ are dependent on the detuning energy, optical pump power, and coupling strength of the system. (B) Valley selective optical stark effect with $\sigma-$ polarization pump, showing the effect on the K valley, but no effect on the $-K\left(K^{\prime}\right)$ valley. (C) Corresponding modification in energy diagram for different valleys with $\sigma-$ pump. The left (right) pair corresponds to the K (-K) valley. (D) Spectral weight transfer (SWT) and energy shift versus fluence/ $\Delta$ (detuning energy). (A-D) Adapted with permission from Macmillan Publishers Ltd., Nature Materials [54], ( $)$ 2014. (E) Optical absorption of two layer WS $_{2}$ with and without an intense laser pump after substraction of a linear background. It shows significant band renormalization and ellimiation of the exciton under high pump power. (F) Schematic of the transition from an excitonic regime to a electronhole plasma with high density of carriers injected by optical pump. (E-F) Adapted with permission from Macmillan Publishers Ltd., Nature Physics [55], (c) 2015.

valleys indicated that the valleys' energy level shifts with circular polarized light pumping. This high magnetic field is difficult to achieve with conventional magnets, yet it can be achieved using femtosecond light pulses [54,56].

Under even higher power incident femtosecond pulses, fluences up to $\mathrm{mJ} / \mathrm{cm}^{2}$, Chernikov et al. [55] showed that the optical response changes over a spectral range of hundreds of $\mathrm{meV}$ in $\mathrm{WS}_{2}$ (Figure 7E). Under an intense pump, a large number of carriers are generated and significantly screen the Coulomb interaction. The sample undergoes a Mott transition from an insulating exciton system to an electron-hole plasma. The absence of excitonic resonances, population inversion, and band gap renormalization of $500 \mathrm{meV}$ confirms the existence of the plasma system (Figure 7F). By monitoring the subsequent dynamics, the pump-induced transition is highly transient with picosecond lifetimes and fully recovers in hundreds of picoseconds [55].

\section{Photonic devices based on 2D excitons}

The light-matter interactions in 2D materials offer several unique advantages in optoelectronics and photonic applications. The strong excitonic resonances and large optical oscillation strength cover light interactions from visible to infrared. In addition, the low dimensionality of 2D semiconductors makes them easily tunable, as the electrical, optical, and mechanical properties can all be controlled using multiple modulation methods. Such flexibility offers potential for device applications such as tunable excitonic optoelectronics devices. The atomically thin vdW nature provides a platform for reducing form factor and substrate-free assembly, compared to semiconductors requiring epitaxial substrates. Finally, most 2D semiconductors have strong emission due to their direct band gap. In this part, we review recent developments in optoelectronic 
and photonic devices based on 2D semiconductors such as excitonic LEDs, optical modulators, integration with photonic and plasmonic structures, and lasers.

\subsection{Excitonic LED}

Now ubiquitous in lighting, LEDs are light sources based on the recombination of electrically injected electrons and holes in $p$ - $n$ junctions. The recombination produces light of color corresponding to the band gap of the semiconductors involved and junction barriers. Currently, LEDs have been widely used for multiple purposes due to the highenergy utilization efficiency, long lifetime, fast switching, and dense packaging. Thus, a naturalistic application for direct band gap 2D semiconductors is in the development of bright, flexible, and ultra-thin LEDs.

In 2013, Sundaram et al. [57] fabricated a Schottky junction between monolayer $\mathrm{MoS}_{2}$ and a $\mathrm{Cr} / \mathrm{Au}$ contact. Applying a large voltage bias $(\sim 2-5 \mathrm{~V})$ in the crystal results in electroluminescence (EL). The authors claim the hot carrier injected under large bias voltages generates excitons at the metal-MoS $\mathrm{S}_{2}$ interface via impact excitation.
The electrically generated excitons recombine to photons in the form of the observed EL [57]. In parallel, Ye et al. [58] achieved exciton-dominant EL in a $\mathrm{MoS}_{2} / \mathrm{Si}$ junction. Further progress enabled the fabrication of in-plane $p-n$ junction on one 2D crystal. By using multiple electrical gates, the TMDC can be tuned to make one side $p$-type and the other side $n$-type (Figure $8 \mathrm{~A}-\mathrm{C}$ ). The in-plane $p$ - $n$ junction provides efficient injection of electron and hole to generate EL with 1000 times smaller injection current [10, 59-61]. In addition, changing the injection current bias can tune the emission from exciton to trion (Figure 8D). Recently, Withers et al. [62] have reported a tunneling LED with an external quantum efficiency of $\sim 10 \%$ at $6 \mathrm{~K}$. Using vertical heterostructures of graphene electrodes, boron nitride tunneling barriers, and $\mathrm{WS}_{2}$, they assemble a fully 2D LED. The vertical junctions reduce the contact resistance, allow for higher current densities, and achieve luminescence from the complete device surface.

As previously mentioned, one major advantage of TMDCs is the control of valley polarization using optical means. However, it is advantageous for devices to utilize an electrical method to achieve this polarization. Zhang et al. [63] demonstrated that a chiral LED can be
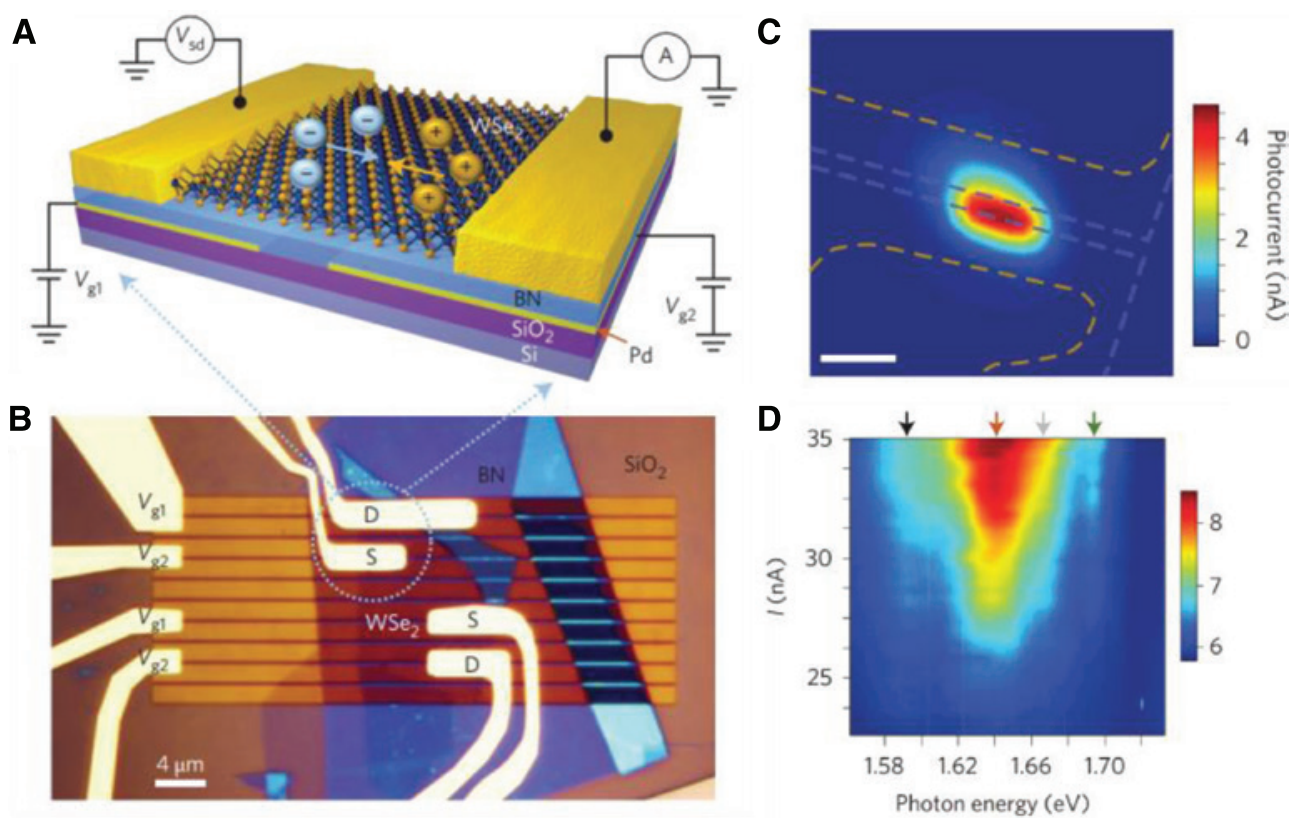

Figure 8: Exciton LEDs.

(A) Schematic and (B) microscope image of monolayer WSe $e_{2}$ LED devices based on $p$ - $n$ junctions. Two palladium back-gates $\left(\mathrm{V}_{\mathrm{g} 1}\right.$ and $\left.\mathrm{V}_{\mathrm{g} 2}\right)$ can separately tune doping levels at corresponding spatial regions to form a $p$ - $n$ junction. Under a source-drain voltage $\left(\mathrm{V}_{\mathrm{sd}}\right)$, the electrons $(\mathrm{blue})$ and holes (yellow) in (A) transport in antiparallel directions and recombine in the junction to yield EL. (C) Scanning photocurrent image at $100 \mathrm{~K}$ shows dominant photocurrent generation mainly in the junction. The orange dashed lines indicate the boundary of source and drain electrodes, while the blue dashed line indicates the boundary of back-gates. (d) Bias current-dependent EL intensity and spectra. The broad spectra include emission from impurity-bound exciton (black arrow), charged exciton (red arrow for $X^{-}$and grey arrow for $X^{+}$), and neutral exciton (green arrow). (A-D) Adapted with permission from Macmillan Publishers Ltd., Nature Nanotechnology [10], () 2014. 
fabricated, emitting $\sigma+$ circularly polarized light under forward bias and $\sigma-$ in the reverse. In TMDCs, lowering the Fermi level into the valence band ( $\sim 80 \mathrm{meV}$ below the edge) results in anisotropic hole behavior, in a phenomenon known as trigonal warping (the conduction band is not as affected). Applying an in-plane bias shifts the electron and hole regions in different directions. This affects the overlap between the electron and holes, which also determines the optical transition strength. Interestingly, under forward and reverse biases, $\pm \mathrm{K}$ valleys are affected differently. They demonstrated that $\mathrm{WSe}_{2}$-based ambipolar devices can form $p-i-n$ junctions that emit circularly polarized EL (Figure 9A,B). The observed valley polarization increases with larger in-plane biases, and up to $20 \%$ is observed for $6 \mathrm{~V}$ at $40 \mathrm{~K}$ and reverses polarization when the bias direction is reversed. However, trigonal warping is related to the crystal's symmetry, indicating that EL is sensitive to the angle between crystal orientation and applied electrical field [63].

Instead of using the valley-dependent electron-hole overlap in trigonal warping, the injection of a spinpolarized carrier into TMDCs can also electrically generate valley polarization. The large spin-orbit coupling in $\mathrm{WS}_{2}(440 \mathrm{meV})$ splits the valence bands to spin-up and
A

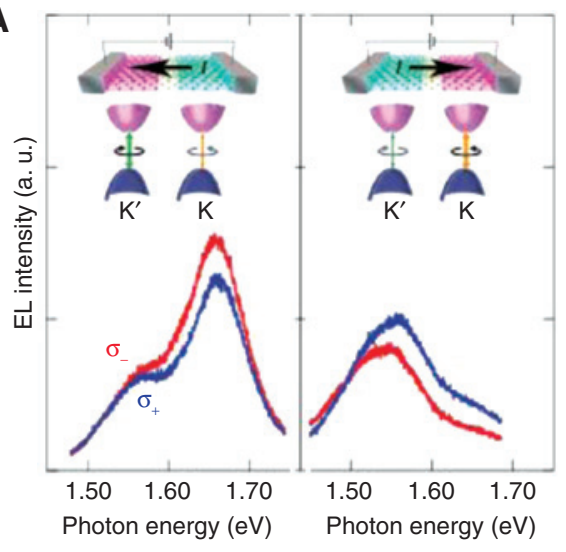

B
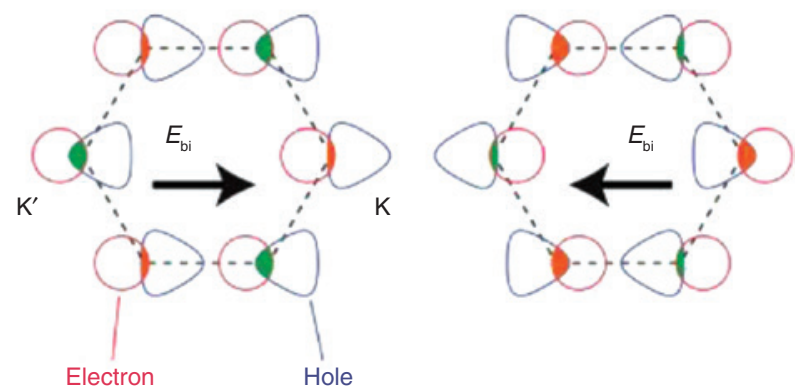

C
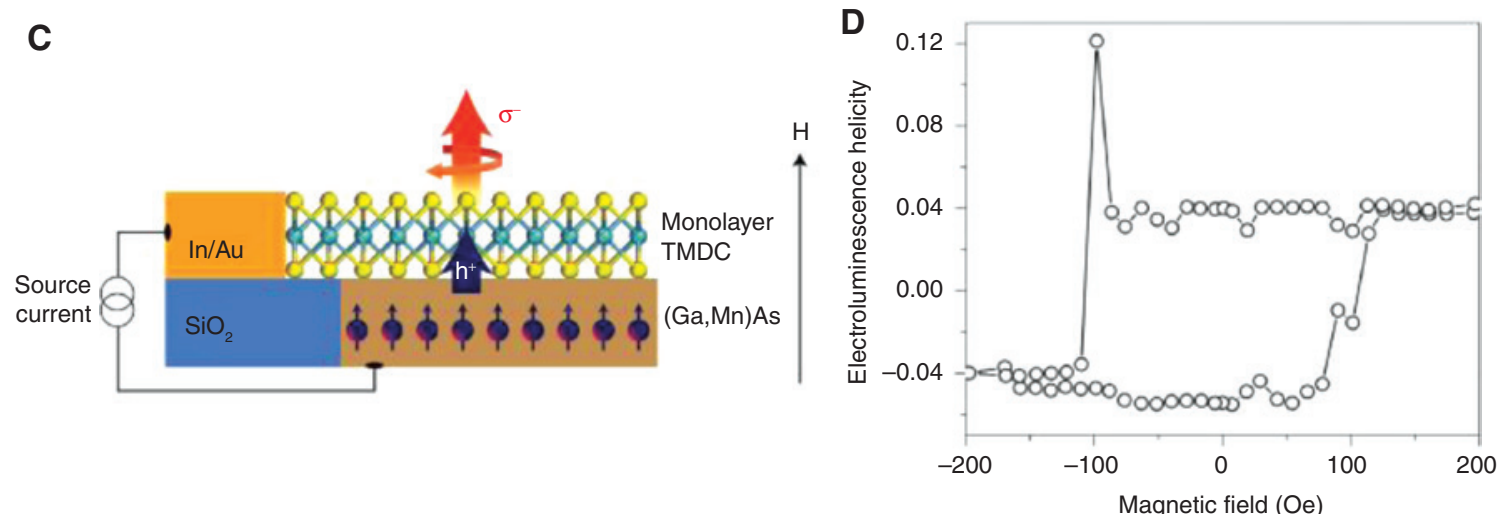

Figure 9: Valley LEDs.

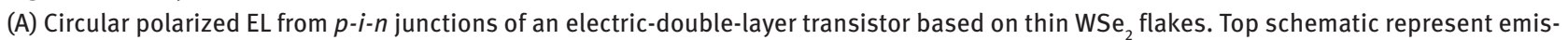
sion intensity difference for $\mathrm{K}$ and $-\mathrm{K}\left(\mathrm{K}^{\prime}\right)$ valley under forward and backward source-drain bias. Bottom spectra are corresponding EL. (B) Illustration of electron-hole overlap shift under a in-plane electrical field in 2D TMDCs with trigonal wraping features (detailed explanation in the text). Red and blue lines outline distribution contours of electron in the conduction band and holes in the valence band. Orange (K) and green areas $(-K)$ show the electron-hole overlap accouting for photon emission of the given polarizations. (A-B) From [63]. Reprinted with permission from AAAS. (C) 2014. (C) Schematic of electrically driven valley polarization via spin injection. (Ga, Mn)As is a ferromagnetic semiconductor used as a spin aligner under external magnetic field. A monolayer TMDC $\left(\mathrm{WS}_{2}\right)$ forms a heterojunction with (Ga, Mn)As and spin-polarized holes can be injected from (Ga, Mn)As to TMDC. The injected holes reside in specific valleys due to spin-valley locking and recombine with electrons in the $n$-type TMDC to emit polarized photons. (D) EL helicity from TMDC as a function of out-of-plane magnetic field. The hysterisis resembles the (Ga, Mn)As magnetization hysterisis loop measured by SQUID. This confirms the efficient spin injection and electrical generation of valley polarization. (C-D) Adapted with permission from Macmillan Publishers Ltd., Nature Nanotechnology [64], (c) 2016. 
spin-down bands. The preservation of time-reversal symmetry requires that bands at the $\pm \mathrm{K}$ valleys must take the opposite spins, forming a spin-valley locking relationship. Using $n$-type $\mathrm{WS}_{2}$, a spin-polarized hole may be injected to selectively recombine with the material's natural electrons. Ye et al. [64] used a dilute ferromagnetic $p$-type semiconductor (Ga,Mn)As to form LED with monolayer $\mathrm{WS}_{2}$. The spins of (Ga, Mn)As, like the bands in $\mathrm{WS}_{2}$, are in the out-of-plane direction (Figure 9C). Applying a forward bias on the (Ga, Mn)As with the WS electrode grounded injects holes across the junction into $\mathrm{WS}_{2}$. The injected holes only populate either the $+\mathrm{K}$ or the $-\mathrm{K}$ valley based on the holes' spin direction (either up or down, controlled by an external magnetic field). As plotted in Figure 9D, the TMDC emission helicity hysteresis as a function of magnetic field is consistent and dependent upon the magnetization hysteresis of (Ga, Mn) As, confirming that valley carriers were generated by spin injection from ( $\mathrm{Ga}, \mathrm{Mn})$ As. The valley-polarized holes recombine with the $\mathrm{WS}_{2}$ electrons to produce $\mathrm{EL}$, with a valley polarization of $16 \%$ at $10 \mathrm{~K}$. Dynamics measurements of the scattering from $+\mathrm{K}$ or $-\mathrm{K}$ can infer a valley injection efficiency of $45 \%$. Injection efficiency can be improved using a cleaner interface and more suitable band alignment between the ferromagnetic semiconductor and the TMDC [64]. Electrical generation of valley carriers can be integrated with manipulation and detection of valley polarization, such as the valley Hall effect, to boost the development of valleytronics.

\subsection{Optical modulator}

Graphene, TMDC, and black phosphorous may meet the emerging demand for compact, efficient, fast, and broadband optical modulators. For example, graphene's zero bandgap enables operation at an extremely broad spectral range extending from the visible to the terahertz frequency due to its unique linear energy-momentum dispersion relation [65]. In addition, low density of states near the Dirac point enables large Fermi level tunability via electrical gate. Meanwhile, high mobility and low resistance allow high-speed electro-optical modulation, up to $\mathrm{GHz}$ [65]. Based on third-order nonlinear processes, saturable absorbers [66, 67], wavelength converters [68], and polarization controllers [69] have been demonstrated with high performance.

Unlike graphene, TMDCs and black phosphorus exhibit direct bandgaps for resonant light absorption, offering properties complementary to graphene. For example, TMDCs typically have large excitonic absorption in the visible and near-infrared, and black phosphorus shows large excitonic absorption in the near- and midinfrared. Compared with graphene, 2D semiconductors have lower mobility and higher resistances and may not be suitable for ultrahigh speed electrical modulation. However, 2D semiconductors can provide larger modulation depth due to their excitonic absorption. In contrast to the universally weak absorption in graphene, 2D semiconductors can absorb more than 10\% of incident light even as a singular layer. In addition, 2D semiconductors support multiple types of external modulation such as electrical, mechanical, optical, and magnetic tuning and show sensitive excitonic response. Furthermore, based on Miller's rule, such strong linear absorption indicates large nonlinear susceptibility for modulation applications such as saturable absorbers. For example, TMDCbased saturable absorbers for all-fiber pulsed lasers in the visible regime have been reported [70], demonstrating the potential for future pulsed fiber laser sources in the visible range. In addition, monolayer TMDCs with inversion symmetry breaking can exhibit strong secondorder nonlinearity. The strength of the generated second harmonic can be electrically controlled and is strongly dependent on the crystallographic orientation of the crystal. With the high optical nonlinearity in 2D semiconductors, more nonlinear frequency conversion processes such as four wave mixing can be applied for light modulation.

\subsection{Purcell effect with photonic and plasmonic structures}

The atomic thickness of 2D TMDCs and strong exciton oscillation strength allow easy integration into and strong coupling with photonic structures such as planar photonic crystal cavities, optical ring resonators, optical microcavities, as well as plasmonic structures. The large binding energy of the excitons indicates a small Bohr radius and large oscillation strength due to the electronhole wave function overlap. As the exciton-photon coupling strength, $g$, is proportional to the square root of the exciton oscillation strength, an enhanced coupling strength is expected for large binding energies.

In the weak coupling regime, where coupling strength $g$ is much smaller compared with exciton linewidth $\Gamma$, the Purcell effect dominates. Namely, by engineering external photonic density of states, the exciton decay rate changes accordingly by the Purcell factor [71, 72]. Such modification has an influence on the PL intensity, valley polarization, and coherence where radiative, non-radiative, and 
inter-valley scattering channels are dynamically linked with each other.

The coupling of 2D TMDCs with optical cavities was first reported by Gan et al. A planar photonic crystal (PPC) nanocavity was designed with a cavity resonance overlapping with the broad 1s exciton resonance in monolayer $\mathrm{MoS}_{2}$. Monolayer $\mathrm{MoS}_{2}$ was transferred onto the cavity after exfoliation on a polymer substrate. Compared with reference samples, five times enhancement of the PL intensity at the cavity resonance wavelengths was observed. Because of the moderate $Q$ factor $(-300)$ and the sub-wavelength mode volume, a large Purcell factor for the cavity mode was deduced to be over 70 [73]. Later, $\mathrm{Wu}$ et al. [74] placed monolayer WSe ${ }_{2}$ onto a photonic crystal $(\mathrm{PhC})$ with a cavity and effectively coupled cavity-mode emission resulting in $\sim 60$ times enhanced PL and a strong pattern of the emission angle in the sub-wavelength scale
(Figure 10A,B). The two planar 2D TMDC-photonic crystal cavity systems allow fabrication for electrical contacts and gate on the monolayer TMDC, providing possibility to electrically manipulate coupling between the cavity photon and 2D exciton. Similar emission enhancements or wavelength selections have been achieved by placing monolayer TMDCs in resonators and microdisk cavities $[76,77]$.

It is also possible to enhance exciton emission by coupling TMDCs to plasmonic structures, such as Ag nanowires (Figure 10C) [75] or Ag disk arrays [78]. The large optical confinement in such structures and strong local field can strongly enhance exciton-plasmon coupling. PL enhancement of about 20 times has been observed (Figure 10D). The strong exciton-plasmon coupling can also induce polaritons, which was studied using angleresolved reflection spectroscopy [79].
A

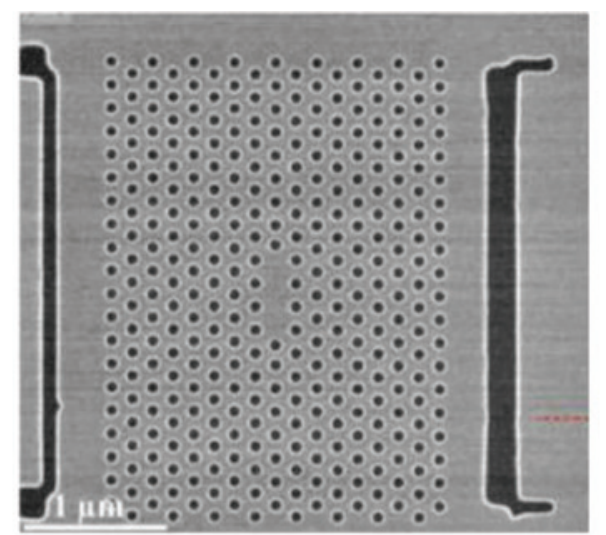

C

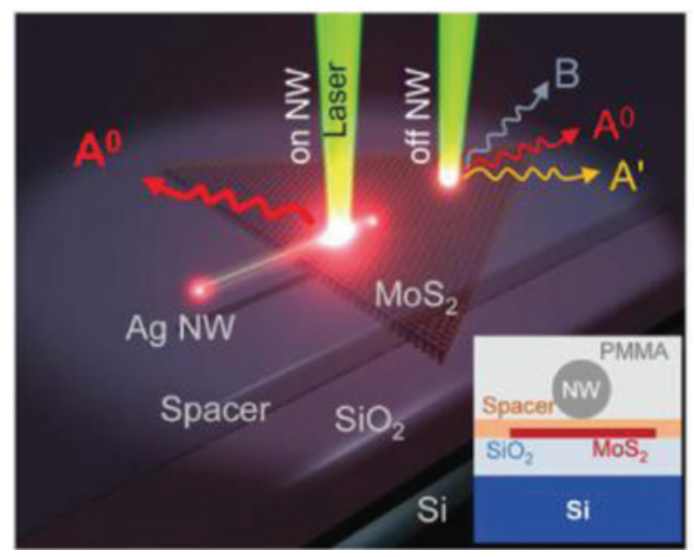

B

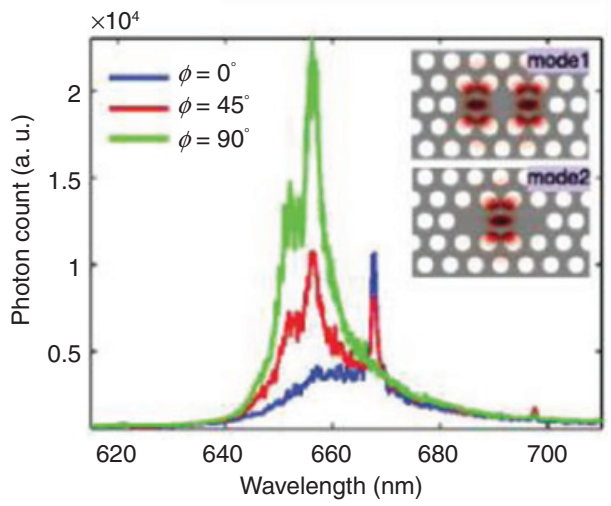

D

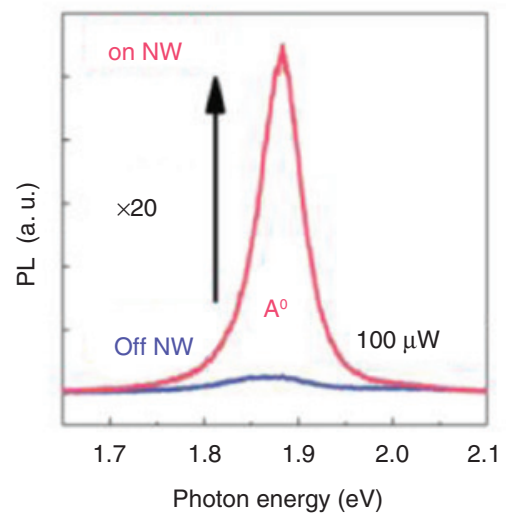

Figure 10: Excitonic enhancements from the Purcell effect.

(A) SEM image of a 2D photonic crystal. (B) Polarization dependence of the cavity-coupled MoS in the cavity. Clearly, a large enhancement can be seen at the correct polarization. (A-B) Reprinted from [73], with the permission of AIP Publishing. (C) 2013. (C) Schematic of hybrid $\mathrm{MoS}_{2}$-Ag nanowire system. (D) PL spectra comparison for $\mathrm{MoS}_{2}$ emission on and off Ag nanowire, showing significant enhancement of the neutral exciton on the Ag nanowire. (C-D) Adapted with permission from [75] (C) 2015 APS. 


\subsection{Exciton-polariton}

In contrast to weak coupling, if $g$ is comparable with or larger than $\Gamma$, the excitons will experience strong coupling. In strong coupling, an exciton is coherently coupled with a photon, resulting in formation of new eigenstates that are half-light, half-matter bosonic quasiparticles called cavity polaritons. The strong light-matter interactions in 2D TMDCs give rise to large $g$, estimated to be tens of meV. Considering the large exciton binding energy (several hundred $\mathrm{meV}$ ), it suggests the possibility of observing the cavity polaritons even at room temperature.

Placing monolayer $\mathrm{MoS}_{2}$ between two distributed Bragg reflector mirrors, Liu et al. [80] observed the first cavity polaritons of $\mathrm{MoS}_{2}$ in a microcavity (Figure 11A). Highly reflective DBRs form a cavity to trap photons, and $\mathrm{MoS}_{2}$ was designed to be placed at the antinode of the mode to have maximum coupling with the photons. The cavity mode for the bare cavity has a moderate $Q$ of $\sim 200$ at room temperature (Figure 11B). As shown in Figure 11C and $\mathrm{D}$, the presence of excitons splits the single resonance cavity mode into two branches in the $E$ - $k$ dispersion with a large Rabi splitting $(\mathrm{h} \Omega \sim 53 \mathrm{meV})$, mapped by angle-resolved reflection and PL spectroscopy [80]. Dufferwiel et al. [81] have further shown the control of detuning between the cavity and the exciton resonances via a microcavity with a tunable cavity length. Depending on the quality of the monolayers and the cavity, the reported value of Rabi splitting varied between 20 and $100 \mathrm{meV}$ [81-83]. As polaritons can retain material properties such as valleys in 2D TMDCs or anisotropic excitons of black phosphorus, they provide a new route to studying more exotic phenomena beyond traditional excitons. In addition, the robust bosonic excitonpolariton with large Rabi splitting observed at room temperature provides the possibility of demonstrating room-temperature polariton condensation at the $2 \mathrm{D}$ limit.

\subsection{Exciton laser}

Strong light-exciton interaction and atomically thin volume are advantages for 2D semiconductors as gain medium for the development of lasers. Multiple cavity designs have been utilized to demonstrate lasing from 2D materials. Wu et al. [84] showed ultralow-threshold lasing below $160 \mathrm{~K}$ in a coupled monolayer $\mathrm{WSe}_{2}$-photonic crystal cavity system $(Q \sim 2500)$. The system was under continuous-wave (CW) pumping and achieved a lasing threshold of $\sim 1 \mathrm{~W} / \mathrm{cm}^{2}$ [84]. In parallel, Ye et al.
[85] reported lasing characteristics such as L-L curve and linewidth narrowing in monolayer $\mathrm{WS}_{2}$ embedded in a high Q (>2000) optical ring resonator. By pumping with a femtosecond pulsed laser at $10 \mathrm{~K}$, a lasing threshold of $\sim 20 \mathrm{MW} / \mathrm{cm}^{2}$ was shown [85]. In a slightly different scheme, Salehzadeh et al. [86] showed lasing from fourlayer $\mathrm{MoS}_{2}$. Oxygen-plasma treated $\mathrm{MoS}_{2}$ acts as a gain medium at the interface between a free-standing microdisk and a microsphere. The $\mathrm{MoS}_{2}$ becomes direct band gap after the plasma treatment and provides additional mode overlap, which enhances the mode overlap and light-matter coupling to reduce the lasing threshold. The L-L curve and line-width narrowing infer a lasing threshold of $>1 \mathrm{~kW} / \mathrm{cm}^{2}$ under CW pumping at room temperature [86].

The discrepancy in the lasing threshold requires additional study to clarify. It may originate from different mode volume overlap and cavity $\mathrm{Q}$ factors or from the different quantum yields in the TMDC samples, which have non-radiative channels such as surface traps and exciton annihilation. As $\mathrm{MoS}_{2}$ can reach a near unity quantum yield PL with chemical treatments, it may be possible to reduce the lasing threshold in the future. Besides optically pumped lasers, electrically controlled lasing devices are needed to develop commercial and integrated devices. Finally, taking advantage of the exotic excitonic physics has a potential to create valley lasers or $\mathrm{THz}$ lasers based on inter-excitonic transitions.

\section{Conclusions and perspective}

In summary, the excitons in 2D materials provide a rich platform for exploring the emerging physics and enable the diverse photonic applications of these materials. The excitons exhibit such a strong effect due to spatial confinement and reduced screening at the 2D limit, where even charged trions are observable at room temperature. Probing the energy of the excitonic series showed that the binding energy of these excitons is extracted into the hundreds of meV. These 2D systems have large oscillator strengths, unique optical selection rules, and versatile tunable parameters. This enables the study of the lightmatter interaction at the molecular thickness limit and inspires rapid progress into developing functional optoelectronics and photonics, such as LEDs and lasers, based on 2D semiconductors.

The current research and understanding of $2 \mathrm{D}$ semiconductors point towards multiple future directions. For example, optical manipulation of the excitonic 
A

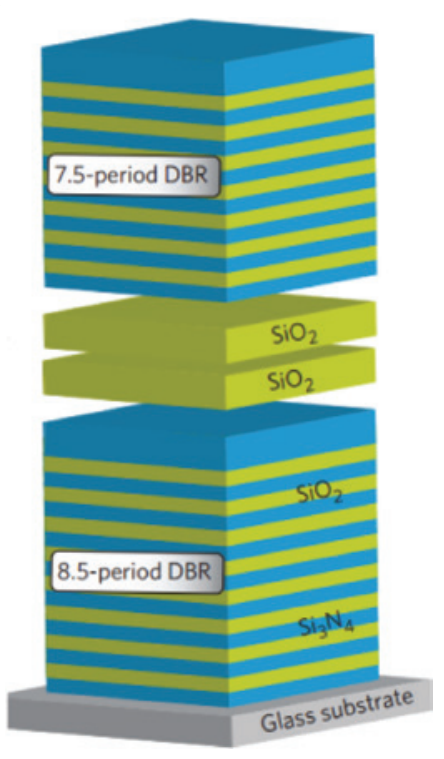

B

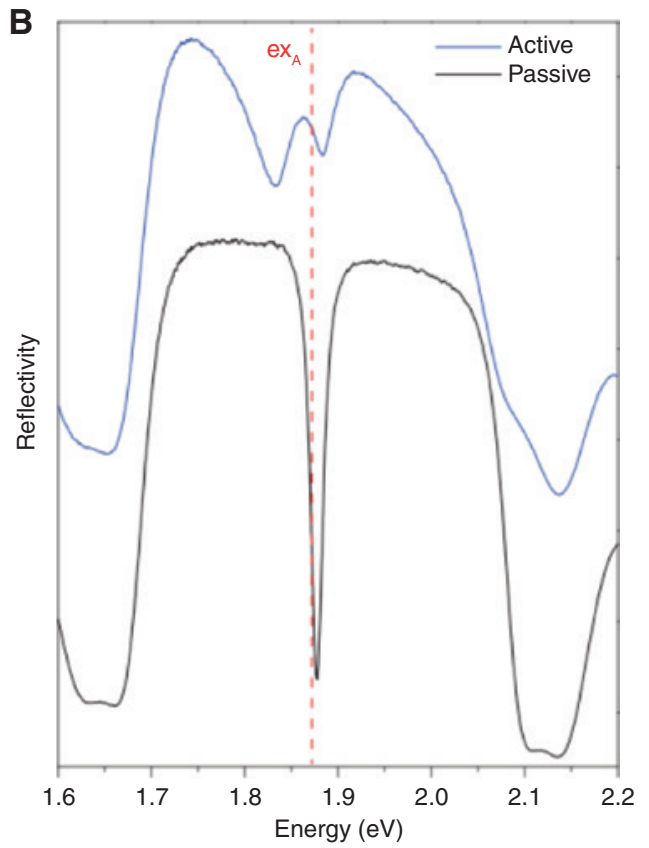

C

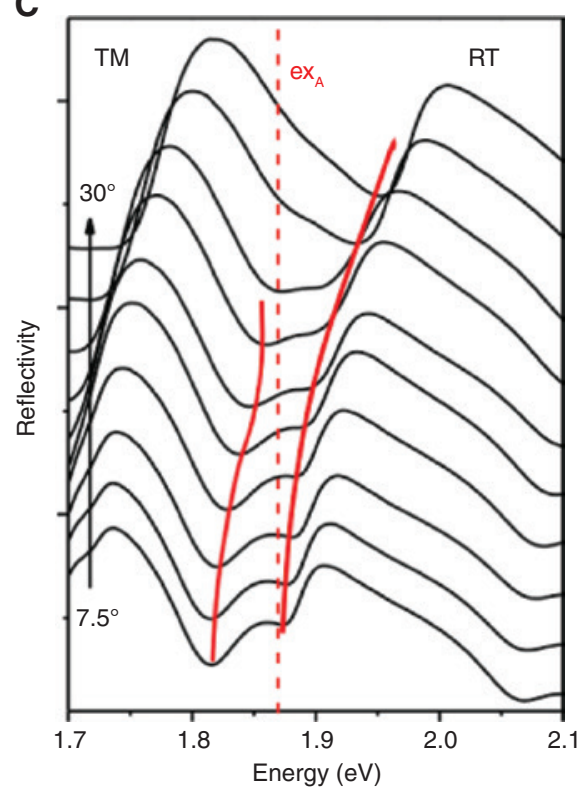

D

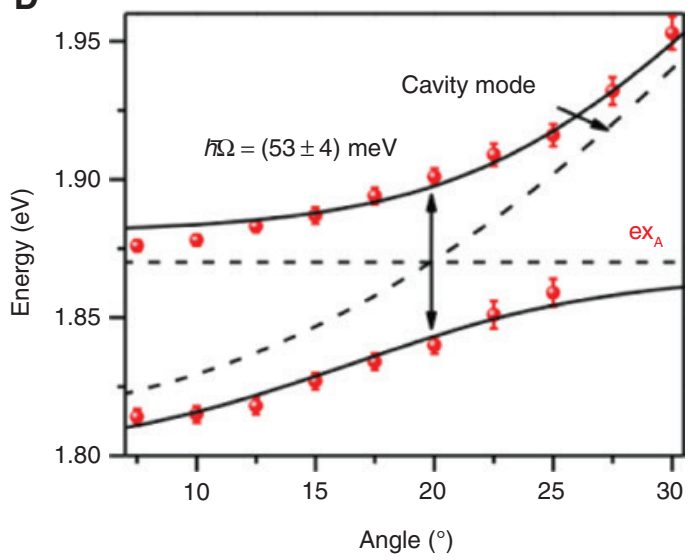

Figure 11: Exciton-polaritons.

(A) Schematic of a typical microcavity using a DBR sturcuture. (B) Reflective spectra for passive location in the microcavity without MoS (black line) and active location with monolayer $\mathrm{MoS}_{2}$ (blue line). Red dashed line indicates the exciton energy. (C) Angle-resolved reflectivity spectra showing strong coupling at room temperature. The red dashed line represents exciton energy without coupling, whereas the red curves show hybrid exciton-polariton modes. (D) Exciton-polariton dispersion. Red dots, showing the energy of exciton-polariton modes, are extracted from red curves in (C). The dashed black lines refer to energy of exciton and cavity mode. The detuing is $-56 \mathrm{meV}$ at zero degrees. A clear Rabi splitting between lower and upper polariton branch is extracted as $53 \pm 4 \mathrm{meV}$. (A-D) Adapted with permission from Macmillan Publishers Ltd., Nature Photonics [80], (c) 2016.

fine structure and utilization of spin and valley have a potential in quantum information. The strong coupling in exciton-polaritons may enable BEC and superfluid behavior in 2D. In addition, the high carrier mobility and infrared band gap in black phosphorus make it ideal for the development of optoelectronic devices. 
Finally, assembling vdW heterostructures can open up a new route for exploring unique exciton physics and applications.

Acknowledgments: This work was supported by the 'Light-Material Interactions in Energy Conversion' Energy Frontier Research Center funded by the U.S. Department of Energy, Office of Science, Office of Basic Energy Sciences under Award Number DE-AC02-05CH11231. M.Z. acknowledges the NSF Graduate Research Fellowship Program (DGE 1106400).

\section{References}

[1] Elliott R. Intensity of optical absorption by excitons. Phys Rev 1957;108:1384.

[2] Feldmann J, Peter G, Göbel E, et al. Linewidth dependence of radiative exciton lifetimes in quantum wells. Phys Rev Lett 1987;59:2337.

[3] Kasprzak J, Richard M, Kundermann S, et al. Bose-Einstein condensation of exciton polaritons. Nature 2006;443: 409-14.

[4] Eisenstein J, MacDonald A. Bose-Einstein condensation of excitons in bilayer electron systems. Nature 2004;432: 691-4.

[5] Miller DA. Optical physics of quantum wells. In: Oppo GL, Barnett SM, Riis E, Wilkinson M, eds. Quantum Dynamics of Simple Systems, London, Institute of Physics, 1996;239-26.

[6] Geim AK, Novoselov KS. The rise of graphene. Nat Mater 2007;6:183-91.

[7] Kang K, Xie S, Huang L, et al. High-mobility three-atom-thick semiconducting films with wafer-scale homogeneity. Nature 2015;520:656-60.

[8] Mak KF, Lee C, Hone J, Shan J, Heinz TF. Atomically thin MoS2: a new direct-gap semiconductor. Phys Rev Lett 2010;105:136805.

[9] Splendiani A, Sun L, Zhang Y, et al. Emerging photoluminescence in monolayer MoS2. Nano Lett 2010;10:1271-5.

[10] Ross J S, Klement P, Jones AM, et al. Electrically tunable excitonic light-emitting diodes based on monolayer WSe2 p-n junctions. Nat Nano 2014;9:268-72.

[11] Ye Z, Cao T, O’Brien K, et al. Probing excitonic dark states in single-layer tungsten disulphide. Nature 2014;513:214-8.

[12] Amani M, Lien D-H, Kiriya D, et al. Near-unity photoluminescence quantum yield in MoS2. Science 2015;350:1065-8.

[13] Mak KF, He K, Shan J, Heinz TF. Control of valley polarization in monolayer MoS2 by optical helicity. Nat Nano 2012;7:494-8.

[14] Schuller JA, Karaveli S, Schiros T, et al. Orientation of luminescent excitons in layered nanomaterials. Nat Nano 2013;8:271-6.

[15] Shi H, Yan R, Bertolazzi S, et al. Exciton dynamics in suspended monolayer and few-layer MoS2 2D crystals. ACS Nano 2013;7:1072-80.

[16] Mak KF, He K, Lee C, et al. Tightly bound trions in monolayer MoS2. Nat Mater 2013;12:207-11.
[17] Esser A, Runge E, Zimmermann R, Langbein W. Photoluminescence and radiative lifetime of trions in GaAs quantum wells. Phys Rev B 2000;62:8232-9.

[18] Kheng K, Cox R, d’Aubigné MY, Bassani F, Saminadayar K, Tatarenko S. Observation of negatively charged excitons $X-$ in semiconductor quantum wells. Phys Rev Lett 1993;71:1752.

[19] Radisavljevic B, Radenovic A, Brivio J, Giacometti i V, Kis A. Single-layer MoS2 transistors. Nat Nanotechnol 2011;6:147-50.

[20] Cheiwchanchamnangij T, Lambrecht WRL. Quasiparticle band structure calculation of monolayer, bilayer, and bulk MoS2. Phys Rev B 2012;85:205302.

[21] Chernikov A, Berkelbach TC, Hill HM, et al. Exciton binding energy and nonhydrogenic rydberg series in monolayer WS2. Phys Rev Lett 2014;113:076802.

[22] Ugeda MM, Bradley AJ, Shi S-F, et al. Giant bandgap renormalization and excitonic effects in a monolayer transition metal dichalcogenide semiconductor. Nat Mater 2014;13:1091-5.

[23] Scholes GD, Rumbles G. Excitons in nanoscale systems. Nat Mater 2006;5:683-96.

[24] Cha S, Sung J H, Sim S, et al. 1s-intraexcitonic dynamics in monolayer MoS2 probed by ultrafast mid-infrared spectroscopy. Nat Commun 2016;7:10768.

[25] Schaibley JR, Yu H, Clark G, et al. Valleytronics in 2D materials. Nat Rev Mater 2016;1:16055.

[26] Ju L, Shi Z, Nair N, et al. Topological valley transport at bilayer graphene domain walls. Nature 2015;520:650-5.

[27] Gunlycke D, White CT. Graphene valley filter using a line defect. Phys Rev Lett 2011;106:136806.

[28] Rycerz A, Tworzydlo J, Beenakker CW]. Valley filter and valley valve in graphene. Nat Phy 2007;3:172-5.

[29] Wu Z, Zhai F, Peeters FM, Xu HQ, Chang K. Valley-dependent Brewster angles and Goos-Hanchen effect in strained graphene. Phys Rev Lett 2011;106:176802.

[30] Xiao D, Yao W, Niu Q. Valley-contrasting physics in graphene: magnetic moment and topological transport. Phys Rev Lett 2007;99:236809.

[31] Xu X, Yao W, Xiao D, Heinz TF. Spin and pseudospins in layered transition metal dichalcogenides. Nat Phy 2014;10:343-50.

[32] Cao T, Wang G, Han W, et al. Valley-selective circular dichroism of monolayer molybdenum disulphide. Nat Commun 2012;3:887.

[33] Xiao J, Ye Z, Wang Y, Zhu H, Wang Y, Zhang X. Nonlinear optical selection rule based on valley-exciton locking in monolayer WS2. Light Sci Appl 2015;4:e366.

[34] Zeng H, Dai J, Yao W, Xiao D, Cui X. Valley polarization in MoS2 monolayers by optical pumping. Nat Nano 2012;7: 490-3.

[35] Yin X, Ye Z, Chenet DA, et al. Edge nonlinear optics on a MoS2 atomic monolayer. Science 2014;344:488-90.

[36] Wang G, Marie X, Gerber I, et al. Giant enhancement of the optical second-harmonic emission of WSe2 monolayers by laser excitation at exciton resonances. Phys Rev Lett 2015;114:097403.

[37] Li L, Yu Y, Ye G], et al. Black phosphorus field-effect transistors. Nat Nano 2014;9:372-7.

[38] Liu H, Neal AT, Zhu Z, et al. Phosphorene: an unexplored 2D semiconductor with a high hole mobility. ACS Nano 2014;8:4033-41. 
[39] Wang X, Jones AM, Seyler KL, et al. Highly anisotropic and robust excitons in monolayer black phosphorus. Nat Nano 2015;10:517-21.

[40] Tran V, Soklaski R, Liang Y, Yang L. Layer-controlled band gap and anisotropic excitons in few-layer black phosphorus. Phys Rev B 2014;89:235319.

[41] Seyler KL, Schaibley JR, Gong P, et al. Electrical control of second-harmonic generation in a WSe2 monolayer transistor. Nat Nanotechnol 2015;10:407-11.

[42] Ross J S, Wu S, Yu H, et al. Electrical control of neutral and charged excitons in a monolayer semiconductor. Nat Commun 2013;4:1474.

[43] Chernikov A, van der Zande AM, Hill HM, et al. Electrical tuning of exciton binding energies in monolayer WS 2. Phys Rev Lett 2015;115:126802.

[44] Santos EJ, Kaxiras E. Electrically driven tuning of the dielectric constant in MoS2 layers. ACS Nano 2013;7:10741-6.

[45] Kim SJ, Choi K, Lee B, Kim Y, Hong BH. Materials for flexible, stretchable electronics: graphene and 2D materials. Annu Rev Mater Res 2015;45:63-84.

[46] Akinwande D, Petrone N, Hone J. Two-dimensional flexible nanoelectronics. Nat Commun 2014;55678.

[47] He K, Poole C, Mak KF, Shan J. Experimental demonstration of continuous electronic structure tuning via strain in atomically thin MoS2. Nano Lett 2013;13:2931-6.

[48] Conley HJ, Wang B, Ziegler JI, Haglund Jr RF, Pantelides ST, Bolotin KI. Bandgap engineering of strained monolayer and bilayer MoS2. Nano Lett 2013;13:3626-30.

[49] Zhu C, Wang G, Liu B, et al. Strain tuning of optical emission energy and polarization in monolayer and bilayer MoS 2. Phys Rev B 2013;88:121301.

[50] Aivazian G, Gong Z, Jones AM, et al. Magnetic control of valley pseudospin in monolayer WSe2. Nat Phy 2015;346:1205-8.

[51] Srivastava A, Sidler M, Allain AV, Lembke DS, Kis A, Imamoğlu A. Valley Zeeman effect in elementary optical excitations of monolayer WSe2. Nat Phy 2015;11:141-7.

[52] Li Y, Ludwig J, Low T, et al. Valley splitting and polarization by the Zeeman effect in monolayer MoSe2. Phys Rev Lett 2014;113:266804.

[53] MacNeill D, Heikes C, Mak KF, et al. Breaking of valley degeneracy by magnetic field in monolayer MoSe2. Phys Rev Lett 2015;114:037401.

[54] Sie EJ, Mclver JW, Lee Y-H, Fu L, Kong J, Gedik N. Valleyselective optical Stark effect in monolayer WS2. Nat Mater 2015;14:290-4.

[55] Chernikov A, Ruppert C, Hill HM, Rigosi AF, Heinz TF. Population inversion and giant bandgap renormalization in atomically thin WS2 layers. Nat Photonics 2015;9:466-70.

[56] Kim J, Hong X, Jin C, et al. Ultrafast generation of pseudomagnetic field for valley excitons in WSe2 monolayers. Science 2014;346:1205-8.

[57] Sundaram R, Engel M, Lombardo A, et al. Electroluminescence in single layer MoS2. Nano Lett 2013;13:1416-21.

[58] Ye Y, Ye Z, Gharghi M, et al. Exciton-dominant electroluminescence from a diode of monolayer MoS2. Appl Phys Lett 2014;104:193508.

[59] Baugher BWH, Churchill HOH, Yang Y, Jarillo-Herrero P. Optoelectronic devices based on electrically tunable $p$-n diodes in a monolayer dichalcogenide. Nat Nano 2014;9:262-7.
[60] Pospischil A, Furchi MM, Mueller T. Solar-energy conversion and light emission in an atomic monolayer $\mathrm{p}$-n diode. Nat Nano 2014;9:257-61.

[61] Lee C-H, Lee G-H, van der Zande AM, et al. Atomically thin $p-n$ junctions with van der Waals heterointerfaces. Nat Nano 2014;9:676-81.

[62] Withers F, Del Pozo-Zamudio 0, Mishchenko A, et al. Lightemitting diodes by band-structure engineering in van der Waals heterostructures. Nat Mater 2015;14:301-6.

[63] Zhang Y, Oka T, Suzuki R, Ye J, Iwasa Y. Electrically switchable chiral light-emitting transistor. Science 2014;344:725-8.

[64] Ye Y, Xiao J, Wang H, et al. Electrical generation and control of the valley carriers in a monolayer transition metal dichalcogenide. Nat Nanotechnol 2016;11:598-602.

[65] Bonaccorso F, Sun Z, Hasan T, Ferrari A. Graphene photonics and optoelectronics. Nat Photonics 2010;4:611-22.

[66] Bao Q, Zhang H, Wang Y, et al. Atomic-layer graphene as a saturable absorber for ultrafast pulsed lasers. Adv Funct Mater 2009;19:3077-83.

[67] Sun Z, Hasan T, Torrisi F, et al. Graphene mode-locked ultrafast laser. ACS Nano 2010;4:803-10.

[68] Hendry E, Hale PJ, Moger J, Savchenko A, Mikhailov S. Coherent nonlinear optical response of graphene. Phys Rev Lett 2010;105:097401.

[69] Bao Q, Zhang H, Wang B, et al. Broadband graphene polarizer. Nat Photonics 2011;5:411-5.

[70] Zhang H, Lu S, Zheng J, et al. Molybdenum disulfide (MoS2) as a broadband saturable absorber for ultra-fast photonics. Optics Express 2014;22:7249-60.

[71] Englund D, Fattal D, Waks E, et al. Controlling the spontaneous emission rate of single quantum dots in a two-dimensional photonic crystal. Phys Rev Lett 2005;95:013904.

[72] Fujita M, Takahashi S, Tanaka Y, Asano T, Noda S. Simultaneous inhibition and redistribution of spontaneous light emission in photonic crystals. Science 2005;308:1296-8.

[73] Gan X, Gao Y, Mak KF, et al. Controlling the spontaneous emission rate of monolayer MoS2 in a photonic crystal nanocavity. Appl Phys Lett 2013;103:181119.

[74] Wu S, Buckley S, Jones AM, et al. Control of two-dimensional excitonic light emission via photonic crystal. 2D Mater 2014;1:011001.

[75] Lee HS, Kim MS, Jin Y, Han GH, Lee YH, Kim J. Selective amplification of the primary exciton in a MoS2 monolayer. Phys Rev Lett 2015;115:226801.

[76] Reed JC, Zhu AY, Zhu H, Yi F, Cubukcu E. Wavelength tunable microdisk cavity light source with a chemically enhanced MoS2 emitter. Nano Lett 2015;15:1967-71.

[77] Yi F, Ren M, Reed JC, et al. Optomechanical enhancement of doubly resonant 2D optical nonlinearity. Nano Lett 2016;16:1631-6.

[78] Butun S, Tongay S, Aydin K. Enhanced light emission from large-area monolayer MoS2 using plasmonic nanodisc arrays. Nano Lett 2015;15:2700-4.

[79] Liu W, Lee B, Naylor CH, et al. Strong exciton-plasmon coupling in MoS2 coupled with plasmonic lattice. Nano Lett 2016;16:1262-9.

[80] Liu X, Galfsky T, Sun Z, et al. Strong light-matter coupling in two-dimensional atomic crystals. Nat Photonics 2015;9: 30-4. 
[81] Dufferwiel S, Schwarz S, Withers F, et al. Exciton-polaritons in van der Waals heterostructures embedded in tunable microcavities. Nat Commun 2015;6:8579.

[82] Flatten LC, He Z, Coles DM, et al. Room-temperature excitonpolaritons with two-dimensional WS2. Sci Rep 2016;6:33134.

[83] Wang S, Li S, Chervy T, et al. Coherent coupling of WS2 monolayers with metallic photonic nanostructures at room temperature. Nano Lett 2016;16:4368-74.
[84] Wu S, Buckley S, Schaibley JR, et al. Monolayer semiconductor nanocavity lasers with ultralow thresholds. Nature 2015;520:69-72.

[85] Ye Y, Wong Z], Lu X, et al. Monolayer excitonic laser. Nat Photonics 2015;9:733-7.

[86] Salehzadeh O, Djavid M, Tran NH, Shih I, Mi Z. Optically pumped two-dimensional MoS2 lasers operating at roomtemperature. Nano Lett 2015;15:5302-6. 